\title{
3 Unicellular Relatives of Animals
}

\author{
Aleksandra Kożyczkowska, Iñaki Ruiz-Trillo and Elena Casacuberta
}

\section{CONTENTS}

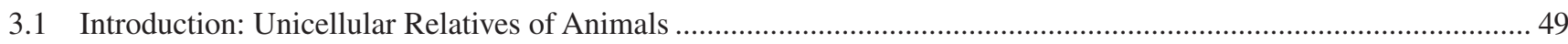

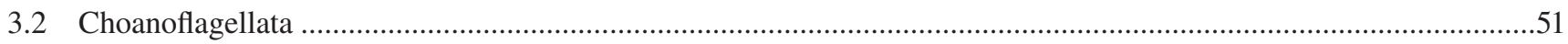

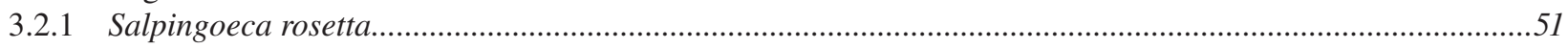

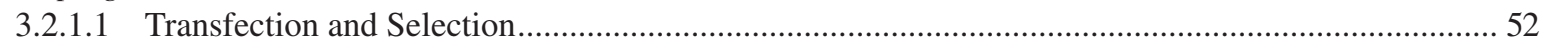

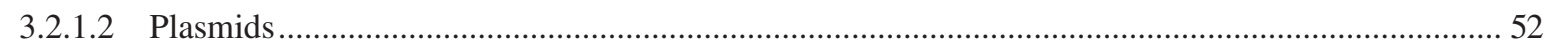

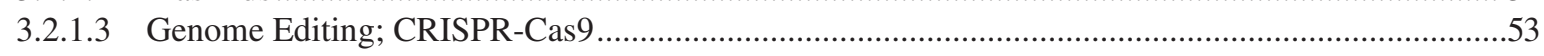

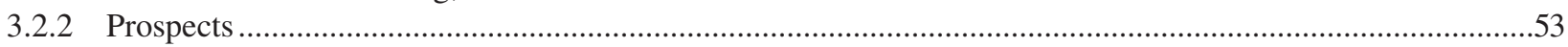

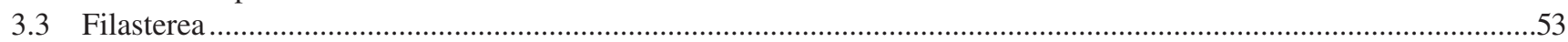

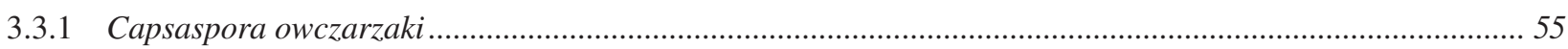

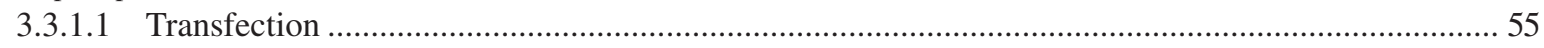

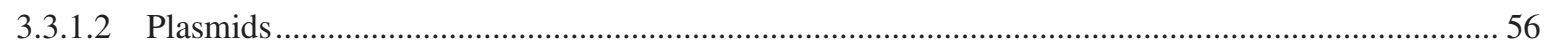

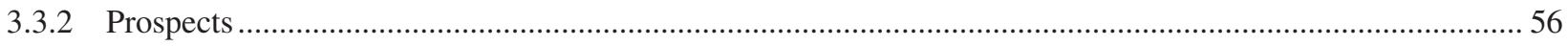

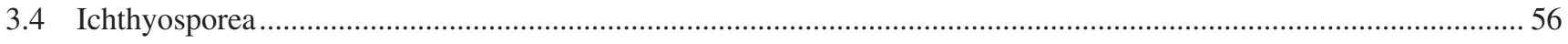

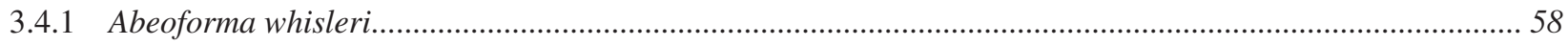

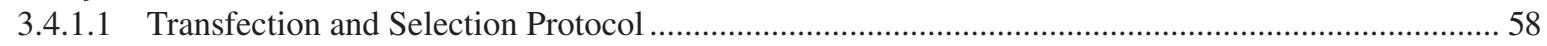

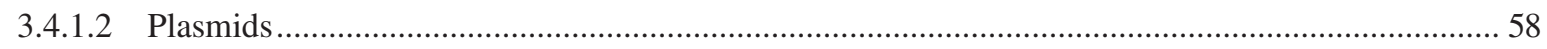

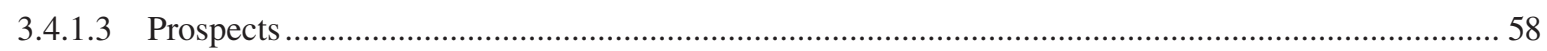

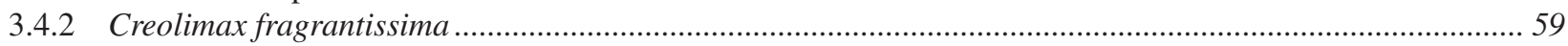

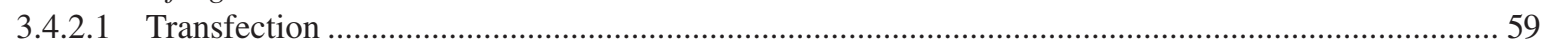

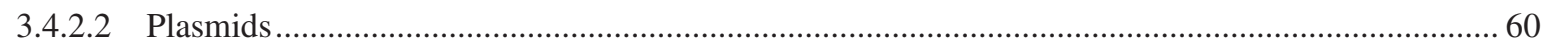

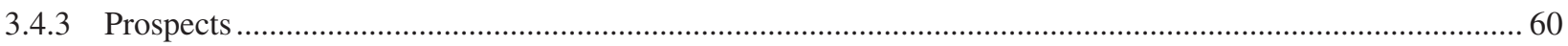

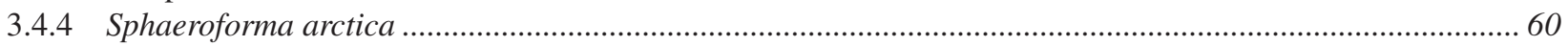

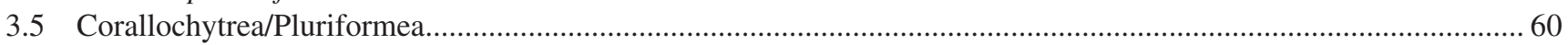

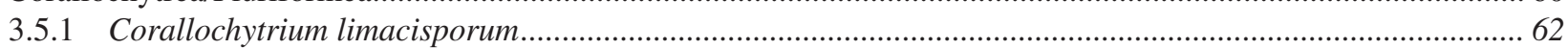

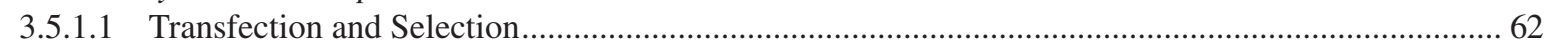

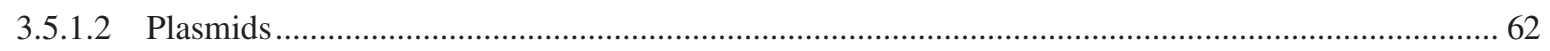

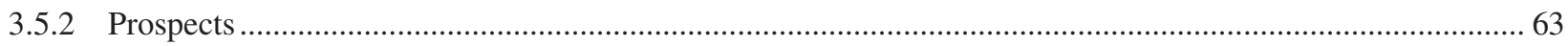

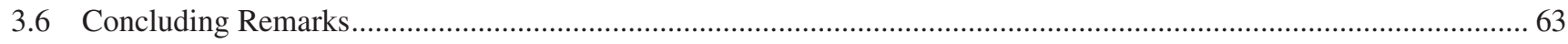

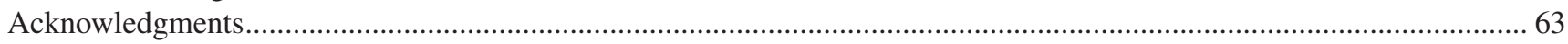

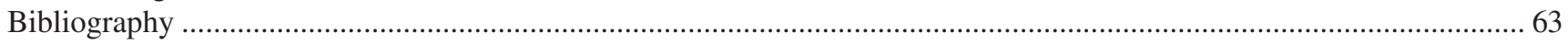

\subsection{INTRODUCTION: UNICELLULAR RELATIVES OF ANIMALS}

All life on Earth has evolved from a common ancestor in a fascinating chain of events. One of the most pivotal steps in the history of life was the transition from protists into multicellular animals. However, how exactly this transition occurred remains unknown. The only way to unveil this process is by studying the unicellular relatives of animals. The Holozoa clade comprises animals and several unicellular lineages (known as unicellular Holozoa): Choanoflagellatea (King 2005), the Filasterea (ShalchianTabrizi et al. 2008), the Ichthyosporea (Mendoza et al. 2002) and the Corallochytrea/Pluriformea (Torruella et al. 2015; Hehenberger et al. 2017) (Figure 3.1).
The analysis of whole genomes from a wide Holozoa taxon sampling in a comparative framework has been useful to reconstruct the genetic content of their common ancestor (Sebé-Pedrós et al. 2017; Grau-Bové et al. 2017; Richter et al. 2018). These phylogenomic efforts have unveiled a unicellular ancestor of animals equipped with a much more complex genetic repertoire than previously thought. One remarkable feature of the ancestor genome is that despite of being unicellular, it already contained many genes whose function is directly related to multicellular structures. Examples of such genes are integrins and cadherins, which are directly related to cell adhesion; tyrosine kinases that mediate signaling in the context of cell-to-cell communication; and several transcription factors involved in development or proliferation such as $\operatorname{runX}, n f-\boldsymbol{\kappa} \beta$ or myc (Abedin and King 2010; Suga 


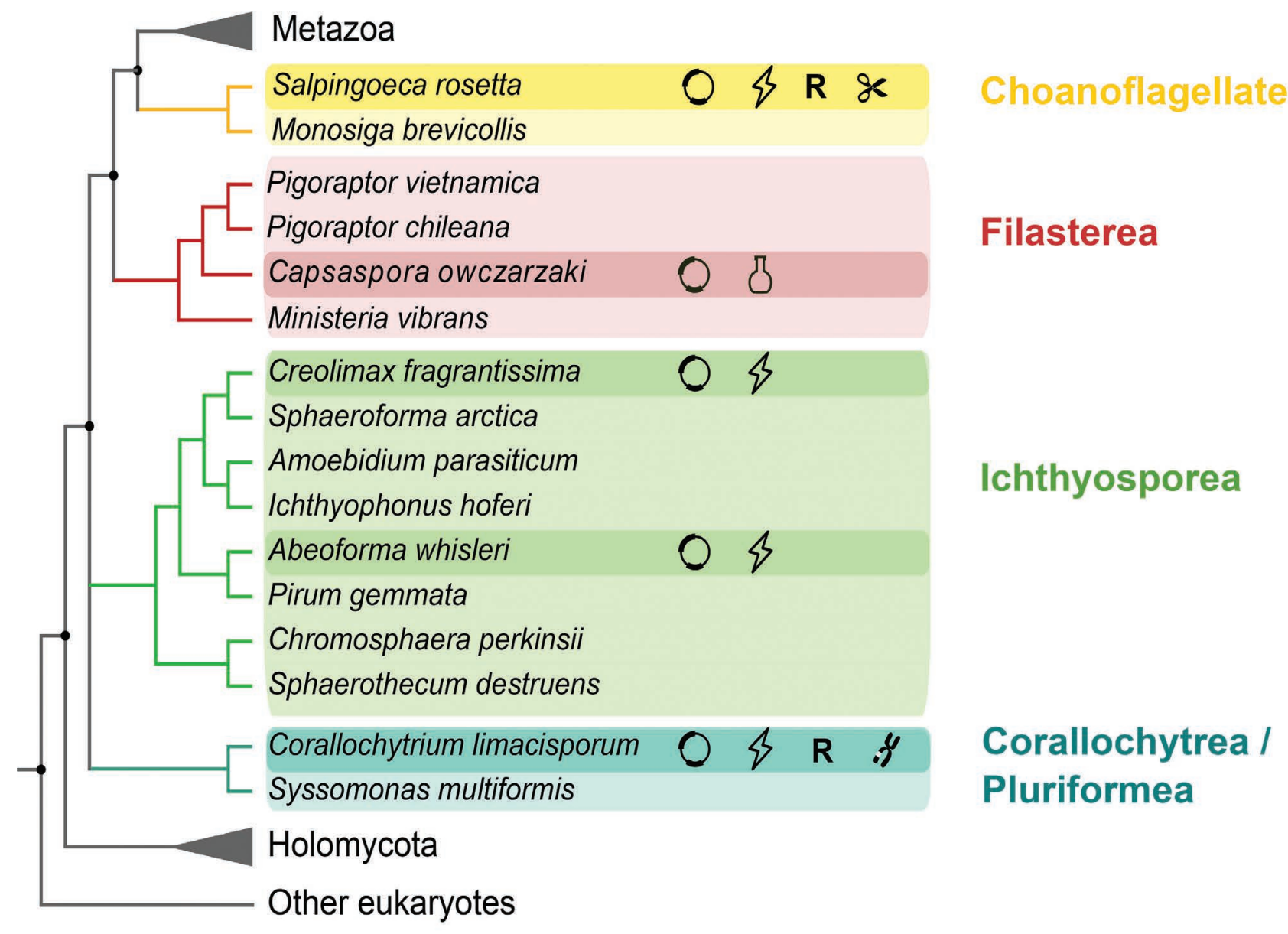

\section{Transfection vector \& Chemical-based transfection \& Electroporation .) Genomic integration \& CRISPR - Cas9}

FIGURE 3.1 Availability of genetic tools for unicellular relatives of animals. Genetic tools are present for each of the lineages of unicellular Holozoa: Salpingoeca rosetta (Choanoflagellatea), Capsaspora owczarzaki (Filasterea), Creolimax fragrantissima and Abeoforma whisleri (Ichthyosporea) and Corallochytrium limacisporum (Corallochytrea/Pluriformea). Symbols represent transfection techniques (electroporation or chemical-based transfection), selection agent, genome editing technique (CRISPR-Cas9) and genome integration. (Phylogenetic tree adapted from Grau-Bové et al. 2017; López-Escardó et al. 2019; Hehenberger et al. 2017.)

et al. 2012; Sebé-Pedrós et al. 2017; Richter et al. 2018). After the initial studies centered in genome content, the next question was to understand if the genome of unicellular holozoans contained some of the features of the regulatory and architectural genome organization observed in Metazoa. Remarkably, genome organization and some epigenetic signatures are present in at least one filasterean, suggesting that they were already present in the genome of the unicellular ancestor (Sebé-Pedrós et al. 2016). Furthermore, since their isolation, different unicellular holozoans have been cultivated, allowing for the first observations and descriptions of some of their stages and cellular characteristics (Marshall et al. 2008; Fairclough et al. 2010; Marshall and Berbee
2011; Sebé-Pedrós et al. 2013, 2017; Torruella et al. 2015; Grau-Bové et al. 2017; Tikhonenkov et al. 2020a). From these studies, we have learned that the four unicellular holozoan lineages are diverse not only in their morphology but also in their developmental modes. Interestingly, in all lineages, there are examples of temporary "multicellular" structures during their life cycle (Figure 3.2). Choanoflagellates are able to form colonies through clonal division (Fairclough et al. 2010; Dayel et al. 2011), the filasterean Capsaspora owczarzaki can form cell aggregation (Sebé-Pedrós et al. 2013) and several ichthyosporeans have a multi-nucleate coenocytic stage that resembles the embryonic coenocyte of some animals (Suga and Ruiz-Trillo 2013a; Ondracka et al. 2018; 
Dudin et al. 2019). Finally, Corallochytrium limacisporum, one of the two representatives of Corallochytrea, combines two different ways to proliferate: through binary fission or through a multi-nucleated coenocyte (Kożyczkowska et al. 2021).

The data generated so far on these unicellular relatives of animals suggest they are key to understanding the evolution from unicellular organisms to multicellular animals. However, we need to go beyond what the genomes tell us and look more particularly at functional analyses, and research efforts in this direction have begun. Genetic tools have been developed for a handful of unicellular holozoans (Figure 3.1), opening the possibility to experimentally test, in a comparative framework, some of the evolutionary hypotheses that the phylogenomic studies have put on the table. In this chapter, we provide a broad description of the general characteristics of each unicellular holozoan lineage, followed by detailed description of the taxa that have been developed into experimentally tractable organisms. We highlight, as well, their particularities and emphasize the most important optimization steps in the different protocols (Figure 3.3). The aim is to provide an updated reference for the state of the art of the methods available for the different unicellular relatives of animals.

\subsection{CHOANOFLAGELLATA}

Choanoflagellates are the sister-group to animals (Figure 3.1). There are around 360 species of choanoflagellates described to date, representing a considerable amount of biodiversity in life forms (King 2005). Choanoflagellates are bacterivorous, and they are commonly found in both freshwater and marine environments (Dolan and Leadbeater 2015). A typical choanoflagellate cell is composed of a single apical flagellum that is surrounded by a collar of microvilli. The currents created by the flagellum help drive bacteria into the collar, where they are phagocytized (Clark 1866; Pettitt et al. 2002). Their morphology and their feeding behavior are also found in the choanocytes, a highly specialized cell type in sponges. These similarities have historically inspired theories of a close evolutionary relationship between animals and choanoflagellates (Clark 1866; Maldonado 2004; Nielsen 2008). However, several phylogenomic analyses point to the fact that these similarities are likely the result of convergent evolution and not shared ancestry (Mah et al. 2014; Sogabe et al. 2019). Phylogenetic analyses divide choanoflagellates in two major clades, Craspedida and Acanthoecida (Carr et al. 2008; Dolan 2015; Paps et al. 2013). Accordingly, both clades show different outer morphologies. In general terms, craspedids form organic coverings which can include a thecate (a vase-like capsule) or a glycocalyx (Leadbeater et al. 2009), and acanthoecids are the species that possess an inorganic extracellular covering made of siliceous material known as the lorica (Carr et al. 2008).

Monosiga brevicollis and Salpingoeca rosetta, both belonging to the Craspedida, are the two better-known choanoflagellates (Figure 3.1) (King et al. 2008; Fairclough et al. 2013). The study of the genome of these two species revealed that they contain genes considered animal specific or involved in multicellular functions, as we will see for other unicellular holozoans (see next sections). Especially intriguing is the presence of synaptic proteins, even though they lack the animal-like mechanism of synapsis (Ryan and Grant 2009; Burkhardt et al. 2014). Those genomes also encode genes involved in forming multicellular structures such as the ones involved in cell adhesion and cell-to-cell communication, such as cadherins or tyrosine-kinase signaling, for example (Hoffmeyer and Burkhardt 2016; Burkhardt et al. 2014). Interestingly, these sets of genes are found in both species independently of their capacity to form multicellular structures, since $S$. rosetta is able to form colonies by clonal division (Figure 3.2a and next section), while $M$. brevicollis is unicellular throughout its life cycle.

Another important result from the study of the genome of $M$. brevicolis and S. rosetta is that they are evolutionarily close, show low genetic diversity and have retained the fewest ancestral gene families in comparison with the other choanoflagellate genomes now available (Richter et al. 2018).

\subsubsection{SalPINGOECA ROSETtA}

So far, efforts to develop a choanoflagellate into an experimentally tractable system have focused on $S$. rosetta. $S$. rosetta presents several advantages among other choanoflagellates to be developed as a new model organism: it has a well-annotated genome and a colonial stage. Moreover, the mechanisms of colonial formation are well understood (Booth et al. 2018; Wetzel et al. 2018; Booth and King 2020). Salpingoeca rosetta, first known as Proterospongia sp., was isolated from a marine sample in the form of a colony (King et al. 2003). The colonies are formed by serial mitotic divisions starting from a single founding cell, which grows into a spherical multicellular structure resembling a rosette (Figure 3.2a) (Fairclough et al. 2010). Interestingly, it has been shown that inside a colony, there are differences between cells concerning their nuclei volume and conformation, the number of mitochondria or cell shapes named afterward chili or carrot cells (Naumann and Burkhardt 2019). These differences among the cells of the colony suggest that there might be spatial cell differentiation in those rosette colonies. Cells inside a rosette seem to hold to each other by cytoplasmic bridges, filopodia and extracellular matrix (ECM; Dayel et al. 2011; Laundon et al. 2019). Although, as mentioned previously, the rosette conformation was the original form in which $S$. rosetta was isolated from the ocean, soon cultured rosettes became infrequent and difficult to control under laboratory conditions, and the single cell became the main form of $S$. rosetta in in vitro cultures. Later, experiments of incubation of $S$. rosetta together with high densities of Algoriphagus machipongonensis, the bacteria with which $S$. rosetta was co-isolated from the ocean, recovered the formation of rosettes. Further investigations discovered that this phenomenon was induced by a lipid, renamed rosette inducing factor (RIF; Alegado et al. 2012; Fairclough et al. 2010; Dayel 
et al. 2011; Woznica et al. 2016). In parallel, a forward genetic screen for mutants unable to form rosettes allowed for the identification of a genetic factor in $S$. rosetta, which could be linked to the rosette phenotype. The recovered rosetteless mutant encoded a C-type lectin and was not able to develop rosettes in spite of being exposed to RIFs (Levin et al. 2014). Although it is not yet fully understood by which molecular mechanism the C-type lectin establishes the relevant interactions, it has been hypothesized that the function of the C-type lectin is related to an interaction with the ECM (Levin et al. 2014). Interestingly, colony formation is not the only stage in $S$. rosetta's life cycle governed by bacteria. For instance, Woznica, Gerdt and collaborators discovered that the bacteria Vibrio fischeri was able to induce sexual behavior in $S$. rosetta through a secreted product that was conveniently labeled EroS (Woznica et al. 2017). Interestingly, EroS was biochemically identified as a chondroitin lyase. This enzyme is able to digest chondroitin sulfate and initiate mating, bearing some similarities to sperm digestion of the egg cover in animal reproduction (Miller and Ax 1990).

Under conditions promoting fast growth, $S$. rosetta is able to form yet another multicellular form different from the rosettes. Linear colonies consist of a chain of cells attached to each other and connected by intercellular bridges and ECM (Figure 3.2a) (Dayel et al. 2011). In the case of single cells, $S$. rosetta can acquire three different forms, which besides its morphology also present a specific behavior: fast swimmers, slow swimmers and thecate cells. The main difference between the different forms of single cell types is the presence of the theca in thecate cells, which consists of a vase-like capsule composed of ECM. All forms of S. rosetta have a flagellum that is used for swimming and orienting the colony, and fast swimmers and rosette colonies also have thin filopodia (Dayel et al. 2011).

Regardless of the availability of genetic tools, $S$. rosetta could already be considered an emerging model system because substantial information on its biology had already been obtained. The rosetteless mutant had been isolated by a forward genetic screen aiming to isolate defective mutants in rosette development (Levin and King 2013; Levin et al. 2014). Moreover, specific culture conditions were developed to obtain and enrich for each of the different life forms of $S$. rosetta (Dayel et al. 2011), and, finally, by the co-cultivation with specific bacteria, mating could be induced (Woznica et al. 2016; Woznica et al. 2017). Nevertheless, tools for direct genetic manipulation, which would allow us for example to fluorescently tag specific proteins to study their localization and dynamics or to knock out target genes, were missing. In recent years, Dr. Nicole King's research group has successfully developed transfection, selection and genome editing for S. rosetta, overcoming these limitations. In the following sections, we will briefly summarize the main steps of these achievements.

\subsubsection{Transfection and Selection}

The transfection protocol for $S$. rosetta is based on the Nucleofection technology, developed by Amaxa (Lonza Cologne AG group) (Figure 3.3a). Nucleofection is a specialized electroporation-based transfection technology engineered to transfer the DNA into the nucleus. This technique proved successful in $S$. rosetta, which can now be transiently transfected with an average efficiency of $1 \%$, similar to what has been achieved in other protists (Janse et al. 2006; Caro et al. 2012).

In order to understand the significance of each optimization step, Booth et al. sequentially eliminated them one at a time and monitored the change in efficiency (Figure 3.3a). For example, the addition of pure and highly concentrated carrier DNA (empty plasmid, such as pUC19), in combination with the plasmid of interest, was key to optimize $S$. rosetta transfection, as observed in other unicellular holozoans (Faktorová et al. 2020; Kożyczkowska et al. 2021). A second key step to boost transfection in $S$. rosetta was priming the cells with a buffer that contains a combination of a protease, a reducing agent, a chelator and a chaotrope (Booth et al. 2018). This specific buffer was key in breaking down the extracellular coat and significantly improved the uptake of transfected DNA into the cell. Even though the extracellular coat is specific for this choanoflagellate, it could be of inspiration for those working on organisms that also possess an extracellular coat or wall, which usually hampers transfection efficiency.

One of the first applications of the developed transfection in $S$. rosetta by Dr. Booth and collaborators was the study of the localization of two septin orthologues, SrSeptin2 and SrSeptin6 (Booth et al. 2018). Septins are a multigenic family involved in highly conserved functions such as cell division (Neufeld and Rubin 1994) but also more specialized functions in multicellular organisms at the level of intracellular junctions and the maintenance of polarity in an epithelium (Spiliotis et al. 2008; Kim et al. 2010). The study of the involvement of septin orthologues of $S$. rosetta in these latter roles can help us understand the contribution of Septins in the evolution of the epithelia before the onset of animals.

Finally, at the same time as the study of Septins in $S$. rosetta, the newly developed transfection technique also proved significant for the characterization of additional rosette defective mutations (Wetzel et al. 2018). In addition, in this study, researchers went one step further by applying selection with the antibiotic puromycin. Selection is very useful in order to enrich the population in a greater proportion of transfected cells Figure 3.3a) (Wetzel et al. 2018). A public protocol for transfection and selection of $S$. rosetta is available at Protocols.io; dx.doi.org/10.17504/protocols. io.h68b9hw

\subsubsection{Plasmids}

As a first step to develop transient transfection, researchers cloned putative endogenous promoters from the elongation factor 1, ef1, $\beta$-actin, act, $\alpha$-tubulin, tub and histone $\mathrm{H3}$ genes from $S$. rosetta. Two different reporter genes, nanoluc (monitored through a luciferase assay) and mwassabi (monitored through expression of green fluorescence), were chosen to test the newly cloned promoters and used to fine-tune the transfection protocol (Booth et al. 2018). 
Besides the battery of transfection plasmids generated to monitor transfection carrying the previously mentioned promoters and reporter genes, researchers engineered plasmids targeting key subcellular structures for future studies on the cell biology of choanoflagellates. With this purpose, they fluorescently tagged the filopodia, cytoskeleton, endoplasmic reticulum, plasma membrane, mitochondria, cytoplasm and nuclei, using specific commercial, highly conserved peptides and protein sequences, known to localize in these cellular compartments (Booth et al. 2018).

Septin orthologues were visualized by the expression of plasmids containing SrSeptin2 and SrSeptin6 fused to the fluorescent reporter mTFP1 (Ai et al. 2006) under the actin promoter.

Finally, from all of the plasmids available for transfection in $S$. rosetta, we want to highlight the possibility of including the puromycin-resistant gene pac in order to select for puromycin-resistant cells (de la Luna S et al. 1988), since wild type $S$. rosetta shows certain susceptibility to this antibiotic (Wetzel et al. 2018).

\subsubsection{Genome Editing; CRISPR-Cas9}

Engineering genome editing from de novo requires not only designing the biochemical strategy that will most likely work in the chosen organism but also, and very importantly, pinpointing a good target. The ideal target should, once being edited in the transfected cells, give a phenotype that would allow further selection of those cells that have been genetically modified; antibiotic resistance or susceptibility is especially useful in this case. To illustrate this concept, we can take as an example the first attempts in genome editing in S. rosetta (Booth and King 2020). The first approach for using the developed CRISPR/Cas9 tools for $S$. rosetta was to introduce a mutation to the rosetteless gene, which had been isolated by a forward genetic screen (see previously) and encodes a C-type lectin protein that is involved in the formation of the rosette phenotype (Levin et al. 2014). The unsuccessful outcome of this first approach was likely due to a low efficiency of the genome editing procedure, which even if it worked correctly could not be detected. A solution to overcome this obstacle is to be able to select the few events of edited cells in the transfected culture by enriching successively in positively transfected cells. Booth and collaborators engineered an alternative CRISPR/Cas9 strategy to confer cycloheximide resistance as an initial step and, in this manner, optimizing the genome editing protocol in $S$. rosetta.

In terms of the molecular reagents needed for CRISPR/ Cas9, the researchers decided to use a ribonucleoprotein (RNP) composed of the expressed Cas9 of Streptomyces pyogenes together with the in vitro-produced single guide RNAs, sgRNA, to direct $S p$ Cas9 to the nicking position. There is a double advantage of using an RNP instead of plasmids for the expression of the different components involved in the editing: on one hand avoiding the necessity of having an endogenous RNA polymerase III promoter in order to express the sgRNAs and on the other avoiding the possible cytotoxicity and off-target problems from uncontrolled Cas9 protein expression (Jacobs et al. 2014; Jiang et al. 2014; Shin et al. 2016; Foster et al. 2018; S. Kim et al. 2014; Liang et al. 2015; Han et al. 2020). Moreover, parallel to transfecting the RNP, a DNA repairing template should be added if the desired mutation is other than a deletion. In the case of $S$. rosetta, Booth and collaborators discovered that $S$. rosetta was able to use a variety of different templates, single and double strand. The addition of the repair template also improved genome editing efficiency. The percentage of genome editing was very similar to transfection efficiency, pinpointing the transfection technique as the limiting factor (Booth and King 2020). Nevertheless, if a good selection strategy exists, the edited cells should be efficiently recovered with this transfection rate with no difficulty.

$S$. rosetta is the first unicellular holozoan to be genome edited. The protocol developed by Dr. Booth and collaborators represents a technical breakthrough that will undoubtedly enhance the possibilities to perform functional studies in this organism. Needless to say, the advances in $S$. rosetta have and will keep inspiring the development of genetic tools and genome editing approaches in other closely related lineages.

\subsubsection{PROSPECTS}

There is no doubt that the technical advances that we have here reported for $S$. rosetta will open new venues to functional approaches that had been hampered until now. We would also like to stress the importance of this organism beyond now being a genetically tractable organism. The importance of $S$. rosetta to address the origin of metazoans has already been broadly explained (Richter et al. 2018). Moreover, the highly organized and structured rosette colonies provide researchers with an ideal model to understand the origins of spatial cell differentiation (Naumann and Burkhardt 2019). Finally, the demonstrated influence of specific interactions with bacteria on essential life events or the transition to multicellular stages of $S$. rosetta provides a unique opportunity to study the interactions between bacteria and eukaryotes (Woznica et al. 2016, 2017).

\subsection{FILASTEREA}

Filasterea is one of the latest lineages of unicellular holozoans that has been described to date. Filasterea is the sister group to Choanoflagellata and Metazoa, all together forming the Filozoa clade (Shalchian-Tabrizi et al. 2008; Torruella et al. 2012, 2015) (Figure 3.1).

There are five species known to belong to Filasterea: Capsaspora owczarzaki, Ministeria vibrans, Pigoraptor vietnamita, Pigoraptor chileana and the recently described and potentially filasterean Tunicaraptor (Figure 3.1) (Owczarzak et al. 1980b; Hehenberger et al. 2017; ParraAcero et al. 2018; Tikhonenkov et al. 2020b). Besides the endosymbiont $C$. owczarzaki, the flagellated species Pigoraptor vietmanita and Pigoraptor chileana are predatory (Hehenberger et al. 2017; Tikhonenkov et al. 2020a), 
(a) Salpingoeca rosetta

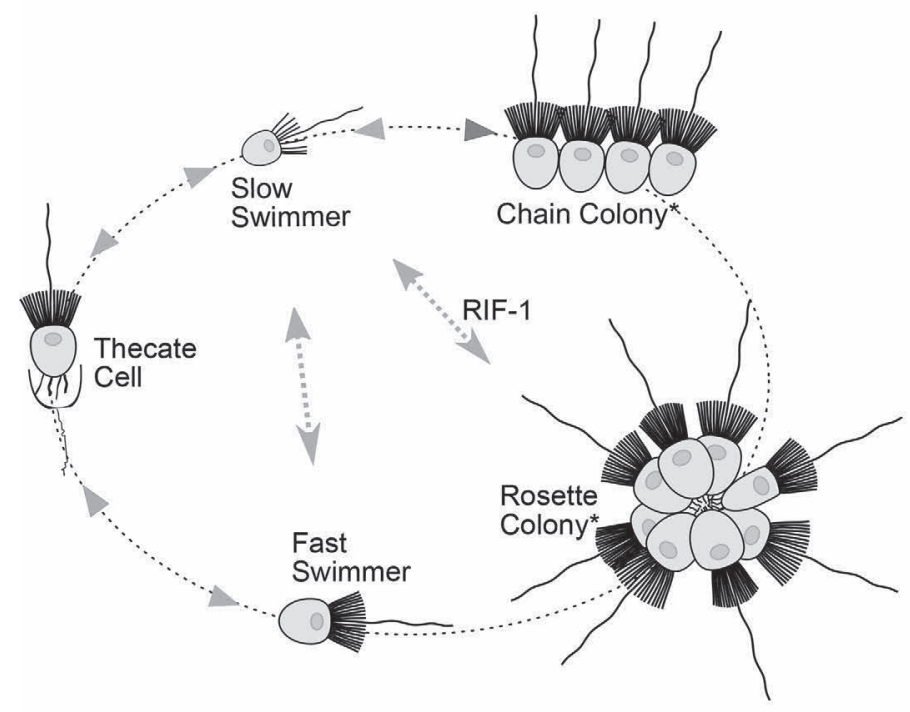

(c) Creolimax fragrantissima

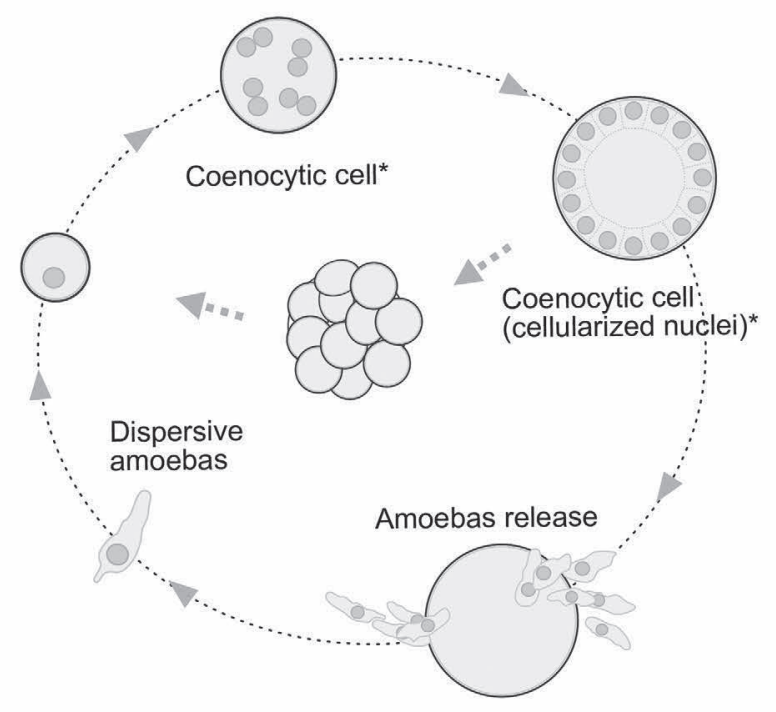

(b) Capsaspora owczarzaki

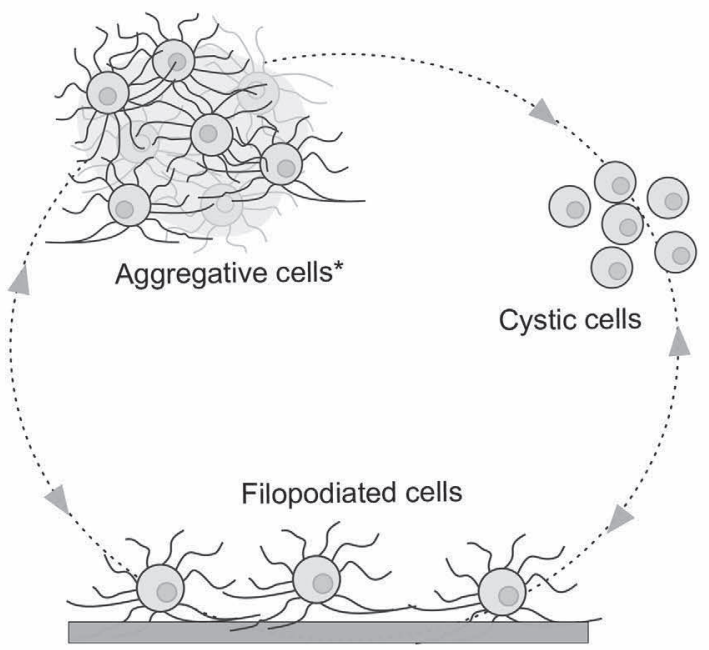

(d) Corallochytrium limacisporum

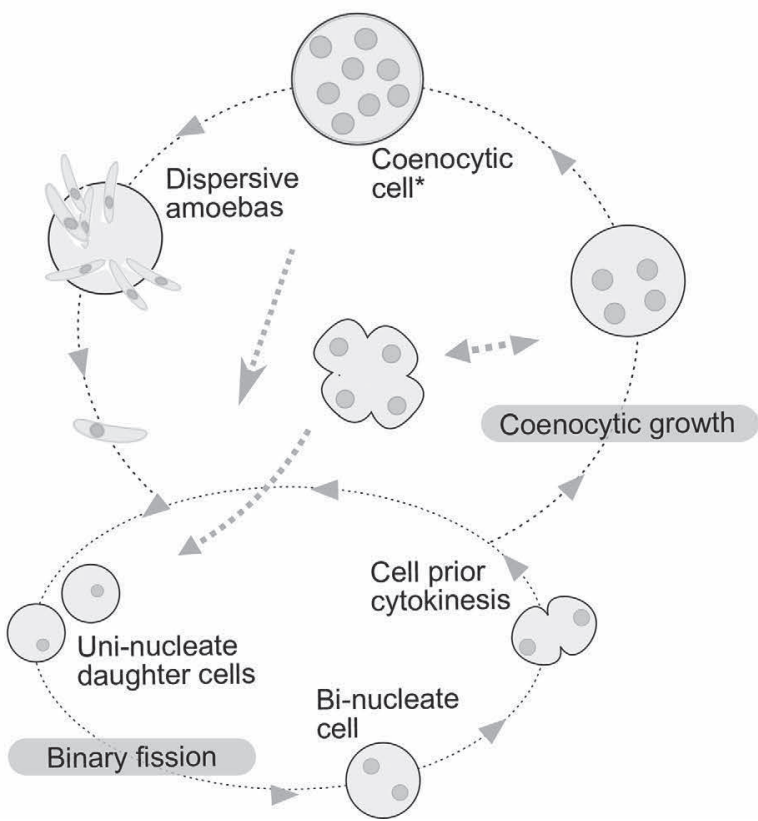

FIGURE 3.2 Models of the life cycle of unicellular relatives of animals. (a) Salpingoeca rosetta, (b) Capsaspora owczarzaki, (c) Creolimax fragrantissima, (d) Corallochytrium limacisporum. Arrows depict observed and inferred transitions between life stages partially described in the main text. Life cycles of unicellular holozoans are diverse but share an important feature: a temporary multicellular-like stage resembling those present in animals (multicellular-like stage indicated with *).

and Ministeria vibrans is a free-living heterotroph (Tong 1997; Cavalier-Smith and Chao 2003; Shalchian-Tabrizi et al. 2008). Filastereans have been isolated from both marine an fresh water environments. For instance, $M$. vibrans has been isolated from samples of marine coastal waters. It has been successfully grown in the laboratory but only in the presence of bacteria, making investigations more difficult. M. vibrans is a spherical amoeboid (aprox. $4 \mu \mathrm{m}$ ) with a stalk falgellum, surrounded by fine and long radiating arms of equal length (Torruella et al. 2015), making a characteristic vibrating movement before attaching to a substrate (Cavalier-Smith and Chao 2003). Interestingly, it has been described that this species is capable of forming aggregative cell clumps (Mylnikov et al. 2019).

Pigoraptor vietnamica and Pigoraptor chileana are two filasteran species isolated from freshwater environments (Hehenberger et al. 2017). Both species have an elongated-oval shape with an average size of 5-14 $\mu \mathrm{m}$ long, have predatory 
behavior and display a very similar life cycle. A detailed description of their complex life cycle can be found in the work done by Tikonhenko et al. (2020a). We would like to highlight that both Pigoraptor species can aggregate during their life cycle, as has been described for M. vibrans as well as for the best-studied filasterean, C. owczarzaki (see the following).

\subsubsection{CAPSASPORA OWCZARZAKI}

First reports of $C$. owczarzaki appeared from investigations on the susceptibility of the fresh-water snail Biomphalaria glabrata to be infected by the parasite Schistosoma mansoni. Studying the possible factors underneath the resistance to infection, Stibbs and collaborators isolated a small amoeba of 3-5 $\mu \mathrm{m}$ in diameter from pericardium and mantle explants from three different strains of B. glabrata, two of them resistant to Schistosoma infection (Stibbs et al. 1979). The ability to grow C. owczarzaki in axenic cultures allowed researchers to test the interaction between the amoeba and the parasite. These works demonstrated that $C$. owczarzaki amoebas were able to adhere to and kill the sporocists of $S$. mansoni, resulting in a high proliferation of C. owczarzaki. H. Stibbs and A. Owczarzaki were the first ones to describe C. owczarzaki and set the initial culture conditions.

The initial stage of the life cycle of $C$. owczarzaki consists of crawling filopodiated amoebas that grow exponentially. Once the culture is saturated and nutrients become limiting, amoebas retract their filopodia and encyst in a round and compact cell, and their growth stabilizes. At this point, encysted cells can attach to each other, forming compact cell aggregates of different sizes (Figure 3.2b). C. owczarzaki cell aggregates can happen spontaneously or can also be induced by agitation with specific parameters (Sebé-Pedrós et al. 2013). Most importantly, electron microscopy analyses revealed that cells in the aggregates are glued together by cohesive extracellular material, which provides the aggregate with consistency but keeps cells individually separated. RNA-seq analyses demonstrated an upregulation of the expression of key genes involved in cell-to-cell communication and cell adhesion, such as the tyrosine kinase signaling pathway and the integrin adhesome (Sebé-Pedrós et al. 2013).

The study of $C$. owczarzaki has not only provided knowledge about its biology but also about the wider question of animal origins. For example, analysis of its genome revealed several genomic features previously thought to be animal specific (Suga et al. 2013; Sebé-Pedrós et al. 2017). C. owczarzaki contains a complete integrin adhesome necessary to mediate the interaction between the cell and the ECM (Suga et al. 2013; Parra-Acero et al. 2020). Moreover, C. owczarzaki also contains a set of proteins, including transcription factors (TFs), known to be involved in developmental pathways in animals; NF- $\kappa b$, Runx and T-box; and others involved in cell motility and proliferation such as Brachyury and MYC (Mendoza and Sebé-Pedrós 2019). Additionally, components of different signal transduction pathways have an unexpected conservation, with examples such as JAK-STAT, Notch, TGF $\beta$ or tyrosine kinases in general (RTKs) (Suga et al. 2012).
It is clear that $C$. owczarzaki was an ideal species to be developed into a genetically tractable organism in order to further investigate the different hypotheses drawn from the genomic content and signatures, as well as to plunge into the terrain of cell biology to enrich the investigations of the evolutionary path shared among holozoans.

\subsubsection{Transfection}

The first attempts to transfect a new organism fail the vast majority of times. For C. owczarzaki, the first protocols to be tested were based on different technologies such as electroporation, magnetofection and lipid-based transfection methods. However, these tests yielded either no positive cells or very low transfection efficiencies, hampering reproducibility (Suga and Ruiz-Trillo 2013; Ensenauer et al. 2011; Parra-Acero et al. 2018). The technology that ended up being efficient enough to be further optimized into a reliable transfection protocol was the classical calcium phosphate precipitation method (Figure 3.2b) (Graham and van der Eb 1973). Here we highlight the steps that turned out to be crucial to improve the efficiency of the transfection protocol (Parra-Acero et al. 2018). One of the factors that is important to maximize efficiency is to use cells at the exponential growth phase. The stage in which C. owczar$z a k i$ is growing exponentially is the adherent stage. Cells from a fresh culture at $90 / 95 \%$ confluence from the adherent stage were the ones with higher transfection efficiency. The size of the crystals from the DNA and the precipitates of calcium phosphate also proved important to improving the efficiency of transfection. The authors determined that the smaller the crystals, the better, as shown for other organisms such as D. discoideum (Jordan and Wurm 2004; Gaudet et al. 2007). In order to achieve a smaller crystal size, it is important to keep the same ratio for DNA/calcium and phosphate when preparing the DNA mix to transfect. The stability of the DNA/calcium ratio once the DNA mix was added to the media also depended on the amount of phosphate in the transfection media, which also needed to be taken into account. Similarly, the $\mathrm{pH}$ of the final solution should be controlled to avoid changes in the solubility of the precipitates. The last touch to further improve transfection efficiency was to expose cells to an osmotic shock, which would permeate the cell membrane for a short period of time. This technique is also used in a variety of eukaryotic cells with the application of glycerol or DMSO (10-20\%) (Grosjean et al. 2006; Gaudet et al. 2007; Guo et al. 2017). In the case of C. owczarzaki, a $10 \%$ glycerol shock during one minute was good enough (Figure 3.3b). Finally, as in any transfection protocol, it is important to be able to identify those cells where the DNA has successfully entered the nucleus and is being expressed. The identification of transfected cells can be done by enriching the transfected population using an antibiotic or a specific drug to which wild type cells (non-transfected cells) are susceptible or by inspecting the expression of a fluorescent protein using fluorescence microscopy. Because C. owczarzaki seems to be resistant to different antibiotics, pesticides or cytostatic 
drugs that are commonly used for selection, the initial plasmids that were designed and transfected into $C$. owczarzaki contained genes encoding small fluorescent proteins. These fluorescent proteins, such as mVenus and mCherry, were expressed in the cytosol of transfected cells. Besides the microscopy observations, efficiency of transfection was also analyzed using flow cytometry by comparing the population of transfected cells with cells from a negative control population. Note that it is important to take into account the possible phenomenon of auto-fluorescence for some types of cells. Efficiency of transfection was on average around $1.132 \% \pm 0.529$ (mean \pm s.d.), which might seem low for researchers working with transfection in other eukaryotic systems, but it is sufficient to efficiently further select transfected cells and proceed with downstream experiments (Parra-Acero et al. 2018).

Co-transfection is known to increase efficiency of the transfection per se, and it is also very useful in order to deliver two different constructs simultaneously. Dr. ParraAcero and collaborators tested in which proportion two different plasmids were uptaken by the cells when co-transfected in order to use co-transfection to visualize simultaneously more than one subcellular structure. Co-transfection resulted, with a rate of incorporation of both constructs almost equally $(72.909 \% \pm 5.468)$ in $C$. owczarzaki (ParraAcero et al. 2018).

Although stable transfection has not yet been developed in Capsaspora, plasmids delivered by transient transfection were shown to be expressed inside the cells for up to ten days. The life cycle of Capsaspora is much shorter than ten days, and therefore this protocol allows for the interrogation of the reporter expression at the different life stages of the organism.

\subsubsection{Plasmids}

The reporter plasmids (pONSY-mVenus and pONSY-mCherry) for optimizing transfection and calculating efficiency were already designed using the endogenous promoter and terminator sequences of the elongation factor 1- $\alpha$ gene (EF1- $\alpha)$ of Capsaspora (Parra-Acero et al. 2018). Besides the engineered plasmids to visualize the cytosol, the researchers went one step further in order to get insights into the cell biology of this species. For this reason, they designed plasmids to fluorescently label the different subcellular structures. For example, the endogenous histone 2B (H2B) gene was fused to mVenus to highlight the nucleus (pONSY-CoH2B:Venus), and the plasma membrane was visualized by cloning the N-myristoylation motif (NMM) of the endogenous Src2 tyrosine kinase gene, which is known to localize at membranes and filopodia (pONSY-CoNMM:mCherry) (Sigal et al. 1994; Parra-Acero et al. 2018). Finally, in order to visualize the cytoskeleton, a small peptide (17 amino acid) named lifeAct known to bind filamentous actin (Riedl et al. 2008) was fused to mCherry (pONSY-Lifeact:mCherry) to visualize the actin cytoskeleton and filopodia of transfected cells. Detailed observations using confocal microscopy of single and co-transfected $C$. owczarzaki cells with these plasmids revealed the targeted structures explaining, among others, the hollow basket structure from the actin bundles around the cell body or the dynamics of the filopodia along the different life stages (Parra-Acero et al. 2018).

\subsubsection{Prospects}

C. owczarzaki, in addition to its key phylogenetic position, its well-annotated genome and the number of "multicellular" genes its genome encodes, is also able to form cell aggregates during its life cycle (Figure 3.2b), making it an ideal organism to analyze the origin of animals.

Finally, the fact that this organism is able to attack and feed on S. mansoni sporocysts (Stibbs et al. 1979; Owczarzak et al. 1980a) also makes it a potential candidate for diseasecontrol strategies, even though the specific interaction of $C$. owczarzaki with the snail B. glabrata remains unclear. Interestingly, $C$. owczarzaki exhibits high resistance to antibiotics and harsh mediums, suggesting its potential in medical applications in the case that was finally selected to control schistomiasis (Parra-Acero et al. 2018).

\subsection{ICHTHYOSPOREA}

Ichthyosporea is the sister-group to Corallochytrea, as well as to the Filozoa (Choanoflagellata, Filasterea and Metazoa) (Mendoza et al. 2002). All described ichthyosporeans are osmotrophs and have multiple life stages that vary greatly in shape and motility and in most cases contain a cell wall of variable composition. The developmental mode of ichthyosporeans is complex and contains multinucleated stages such as a coenocyte (Figures 3.1 and 3.2c).

Ichthyosporeans received this name because the early identified representatives were all parasites of fish (CavalierSmith 1998). Later phylogenomic analyses of rDNA with newer representatives expanded the group in two internal classes, the Dermocystida, which are exclusively parasites of vertebrate hosts, and the Ichthyophonida, which can parasitize a variety of host species (Mendoza et al. 2002; Marshall et al. 2008). In accordance with their habitat, only representatives of Ichthyophonida can be cultured in laboratory conditions (Jøstensen et al. 2002; Marshall et al. 2008). Interestingly, the motile representatives of Dermocystida are equipped with a flagellum, while the ichthyophonids are motile amoebas. Maybe related, it has been shown by electron microscopy studies that representatives of Ichthyophonida have a spindle pole body (Marshall et al. 2008), which would nicely correlate with the disappearance of centrioles and the flagellum as a consequence (Marshall and Berbee 2011). On the other hand, centrioles have been described for members of Dermocystida such as Dermocystidum percae (Pekkarinen 2003). In the coming years, further investigations on other key biological questions will be possible once experimentally tractable organisms will be developed for both subclasses. For instance, investigations on the microtubule organizing centers and the nature of the mitosis (whether it is open, closed or somewhere in between) would 


\section{Salpingoeca rosetta, 2018}

Nucleofection Lonza ${ }^{\circledR}$

Stable

transfection
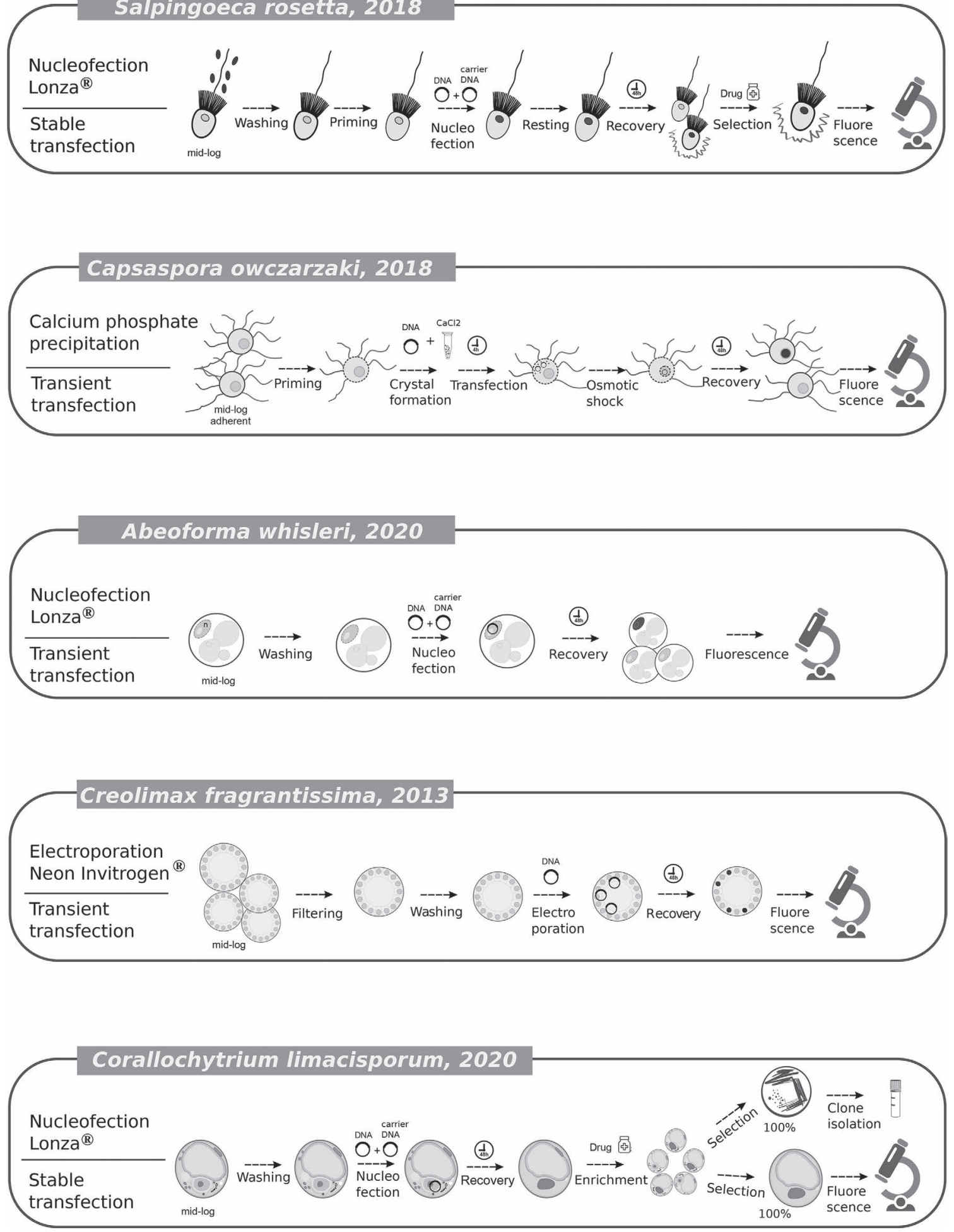

FIGURE 3.3 Schematic diagram of transfection protocols among unicellular relatives of animals. Basic steps have been illustrated. Key steps for electroporation-based techniques: pre-washing the remaining growth medium and addition of carrier DNA to the DNA of interest; for S. rosetta and C. owczarzaki, cells are primed for a higher membrane permeability. For calcium phosphate protocol: crystal size formation (ratio of $\mathrm{DNA} / \mathrm{CaCl}_{2}$ ) and an osmotic shock. For each transfection protocol, cells have been at the exponential growth phase (mid-log). Drug selection and stable transfection have been achieved in two organisms: Salpingoeca rosetta and Corallochytrium limacisporum. Additionally, C. limacisporum can be grown on an agar plate, allowing for single clone isolation. 
be of great interest and could provide further insights on the evolutionary history of both subclasses.

\subsubsection{ABEOFORMA WHISLERI}

A. whisleri was isolated from the digestive track of the filtering mussel Mytilus (Figure 3.1) (Marshall and Berbee 2011). In culture, A. whisleri grows axenically in artificial Marine Broth (MB; GIBCO) at $13^{\circ} \mathrm{C}$. Cultures can be seeded at low density $10^{4} / \mathrm{mL}$ and reach confluence in approximately two weeks.

A. whisleri presents a vast myriad of cell shapes, which makes it difficult to reconstruct a possible life cycle from simple optical microscope observations. In a regular $A$. whisleri culture, one can observe mobile amoebas of different shapes, hypha-like stages, plasmodia cell shape, cells of different length and bigger and rounder multinucleated cells that correspond to coenocytes. Through live observations, researchers have witnessed the release of amoebas from the rounded coenocytic cells as well as vegetative reproduction, which can take place from sporadic budding of the plasmodium. For a thorough description of different cell shapes of A. whisleri, see Marshall and Berbee (2011).

All forms of A. whisleri cells are quite delicate even though it has been reported that all of them have a cell wall (Marshall and Berbee 2011). Interestingly, embedded membrane-bound microtubules (MBTs) were described for several of the morphologically different forms of $A$. whisleri cells. MBTs could be instrumental for equipping $A$. whisleri with the high membrane flexibility that it exhibits while having a cell wall. This could also be the reason behind the strong sensitivity that $A$. whisleri cells show when confronted with chemical, physical or electric shocks to create membrane pores in order to achieve transfection.

\subsubsection{Transfection and Selection Protocol}

One of the first steps toward developing genetic tools in $A$. whisleri was to test a wide battery of drugs for susceptibility in order to identify a selective agent (Faktorová et al. 2020). Puromycin resulted in the most promising acting as a cytostatic agent when assayed between 100 and 500 micrograms $/ \mathrm{mL}$, opening the possibility to use the resistance gene for puromycin activity ( $p a c$ ) (Luna et al. 1988) and the following protocol at Protocols.io: www.protocols.io/view/ testing-selective-agents-for-the-icthyosporeans-ab-z5nf85e).

To achieve insertion of DNA inside A. whisleri nuclei, a battery of transfection protocols based on different methods were tested. Initially, electroporation with the Neon electroporation system (Invitrogen) was successful, but the resulting efficiency and reproducibility of this protocol did not allow for a regular establishment of transfection. During this time, researchers working on the choanoflagellate $S$. rosetta achieved promising results with another electroporation-based system, Nucleofection (Lonza), which was also more efficient and reproducible for A. whisleri (Figure 3.3c) (Booth et al. 2018; Faktorová et al. 2020; and Protocols.io: www.protocols.io/ view/abeoforma-whisleri-transient-transfection-protocolzexf3fn). In summary, the key steps to significantly improve efficiency and reproducibility were as follows: washing the cells with 1X PBS - which should be completely eliminated prior to re-suspension with transfection buffer-was important to maintain the low salt concentration for applying the electric shock. Small variations in this sense would make A. whisleri cells very susceptible to electric shock, exploding easily. On the other hand, immediate re-suspension of the cells with MB after the application of the electric current was key to obtaining the best cell recovery possible. The addition of high-concentration and high-quality carrier DNA (empty $p U C 19$ ) was key to increasing the number of transfectants up to an order of magnitude. Finally, the best parameters for transfection were the combination of the buffer P3 in the middle of the scale of stringency and the electroporation code EN-138 (all provided by Lonza) (Figure 3.3c). After $24 \mathrm{~h}, \sim 1 \%$ of the culture was transformed based on the fraction of cells expressing mVFP (venus fluorescent protein) in the nucleus.

As an example of successful transient transfection for A. whisleri, Figure 3.4a shows the result of transfecting AwH2BmVenusTer. Several positive cells were observed with specific mVenus expression in the nuclei, demonstrating that the AwH2BmVenusTer plasmid was correctly delivered. Nevertheless, cells did not progress with cell division, suggesting that the expression of the fusion protein mVenus$\mathrm{H} 2 \mathrm{~B}$ might be excessive, thus making the cells susceptible to the high levels of histone protein (Singh et al. 2010).

\subsubsection{Plasmids}

In order to deliver exogenous DNA into A. whisleri with the possibility to obtain transcription and protein expression, constructs with fluorescent proteins such as mCherry and mVenus (Shaner et al. 2004) (Nagai et al. 2002) were engineered using endogenous promoters to drive transcription. The actin promoter was chosen as one of the constitutive promoters widely used in molecular biology and therefore likely to work. Signatures from endogenous genes were selected in order to drive the fluorescence to a subcellular structure that could be easily identified, such as the nucleus (AwH2BmVenusTer) (Figure 3.4a) or the cytoskeleton (ApmCherryTubulinaTer, ApmCherry Actina Ter), all under the A. whisleri actin promoter and terminator (Faktorová et al. 2020). Moreover, a construct from which puromycin resistance could be delivered was also engineered in order to achieve stable transfected lines in the future (ApmCherryPuromycinaTer).

\subsubsection{Prospects}

In the near future, combined efforts to achieve stable transfection in A. whisleri under the effect of puromycin, together with simultaneously improving transient transfection toxicity, will be implemented. Because of the rich complexity in morphology of A. whisleri cells, achieving stable transfected lines with differently labeled subcellular components will be instrumental to study the sequence and diversity of its 
life stages and to be able to reconstruct its life cycle and the regulation of their transition.

\subsubsection{Creolimax fragrantissima}

C. fragrantissima was the first unicellular holozoan to be transiently transfected (Suga and Ruiz-Trillo 2013a), and it is so far the ichthyosporean with the greatest aptitude for being turned into a model organism (Figure 3.1). Most importantly, $C$. fragrantissima has been isolated a considerable number of times, and most of them have been successfully cultured in the laboratory. Besides having been isolated from a myriad of invertebrates belonging to four different phyla, the isolated $C$. fragrantissima strains were highly similar at both the molecular and morphological level (Marshall et al. 2008). The observed uniformity of the different strains implies relevance of the obtained results for a wide range of organisms, which is definitely desirable for a model organism.

C. fragrantissima is an osmotroph organism with an apparent asexual linear life cycle (Figure 3.2c). Cells are small and round, uni- or bi-nucleated, with a smooth cell wall and central vacuole, which pushes the nuclei to the cell periphery. There is no sign of flagella, hypha or budding behavior. The round cell grows from $6-8 \mathrm{~mm}$ in diameter to a mature multinucleated coenocyte of $30-70 \mathrm{~mm}$ in diameter, from which motile amoebas will burst from several pores of the parental coenocyte wall. Crawling uni-nucleated amoebas $12 \mathrm{~mm}$ long and 4.5-5 $\mathrm{mm}$ wide with erratic movement will become round and encyst after exploring a certain distance in various directions and finally setting, becoming round cells again, the cysts (Suga and Ruiz-Trillo 2013a; Marshall et al. 2008). The release of already round encysted cells has also been documented, as well as endospores that manage to grow without ever exiting the parental cell (Marshall et al. 2008). Fusion of cells is not observed, although clumps of cysts getting together are often found in regular cultures. The whole life cycle takes about 44 hours, where the maturation of the amoebas inside the coenocyte corresponds to 2-3 hours (Figure 3.2c).

\subsubsection{Transfection}

C. fragrantissima was the first unicellular holozoan in which transient transfection was achieved, allowing for the first investigations on its life cycle and initial characterization of life stages at the cellular level (Suga and Ruiz-Trillo 2013). Moreover, C. fragrantissima is the only unicellular holozoan for which morpholino RNA silencing has been successful (Suga and Ruiz-Trillo 2013).

The initial transformation protocol was based on electroporation performed inside the solution of the cell suspension using a wire-type electrode (Kim et al. 2008). With this protocol, the authors reported a remarkable transfection efficiency of 7\% (Suga and Ruiz-Trillo 2013). Despite the transfection being transient, the introduced plasmid allowed for expression of the tagged protein during a two-day period. This was sufficient for the plasmid to be passed on to the next generation, enabling for the first time the description of some of the life stages of C. fragrantissima. The authors of the study specifically labeled the nuclei by fusing the $H 2 B$ gene of either $C$. fragrantissima or the close relative Sphaeroforma arctica (Figure 3.1) with a fluorescent protein mCherry (see Figure $3.4 \mathrm{~b}$ for an example of $C$. fragrantissima transfected with an equivalent plasmid specifically expressing mVenus in the nuclei of a coenocyte). These positively transfected cells allowed researchers to determine through time-lapse experiments the synchronicity of the nuclear divisions in the C. fragrantissima coenocytes.

These first transformation experiments in C. fragrantissima also opened the door to the possible direct manipulation of the organism by performing gene silencing. In the scenario where no transgenic organisms can be engineered, the alternative to transient gene silencing by either interfering with transcription or translation with antisense RNA matching the right targets can be an alternative functional approach. The fact that the cell wall of $C$. fragrantissima seems to be the thinnest and least complex of the known ichthyosporeans might have facilitated the success of this approach (Marshall et al. 2008). The authors chose morpholinos (i.e. synthetic small interfering RNAs, or siRNAs) to proceed with gene silencing of the transformed recombinant proteins. Because the effect of silencing was directly related to the efficiency of the transfection, an internal control needed to be established. For this reason, the authors first obtained the correlation between the intensities of the different fluorescent markers mCherry and mVenus. The transfections always proceeded with the corresponding antisense RNA targeting the gene of interest fused to mCherry together with a plasmid that expressed the cytoplasm fluorescent marker (mVenus). The decrease in mCherry fluorescence compared with the main intensity of the mVenus would give the percentage of achieved silencing. By repeating the experiments with siRNAs containing mismatches as a control, the authors were able to demonstrate that their functional RNAi approach was specific (three mismatches were enough to abolish the silencing effect on the mCherry expression). Interestingly, the authors also demonstrated that the silencing effect could be achieved by using this transfection method to block translation. In this case, the antisense RNA was directed to the 5'UTR region of one of the constructs. The results were similar, but in this case, five mismatches were necessary to lose sequence specificity (Suga and Ruiz-Trillo 2013a).

Further steps on the development of genetic tools in $C$. fragrantissima have been hampered by the lack of a suitable selective agent with a known resistance gene to achieve stable transfection. We and other researchers are working on this matter in order to be able to genetically modify $C$. fragrantissima. Previous research on this organism has unveiled a number of undoubtedly interesting avenues that will be possible to investigate after the development of more advanced genetic tools. 


\subsubsection{Plasmids}

The expression cassettes reporting transfection were constructed using the endogenous $\beta$-tubulin promoter of $C$. fragrantissima to drive expression of a fluorescent protein, either mCherry or mVenus. For nuclei labeling, the cassette fused the mCherry fluorescent protein to the endogenous histone $2 B(\mathrm{H} 2 \mathrm{~B})$ gene of $C$. fragrantissima. Interestingly, a fusion to the $S$. arctica $h 2 B$ gene was also functional in C. fragrantissima. For cytoplasm labeling, the authors co-transfected the H2B-mCherry construct with a vector expressing the mVenus fluorescent protein driven by the same $\beta$-tubulin promoter from $C$. fragrantissima (Suga and Ruiz-Trillo 2013).

\subsubsection{Prospects}

Interestingly, for both $C$. fragrantissima and also for $S$. arctica (see the following), a subset of long non-coding RNAs are specifically regulated for some life stages (de Mendoza et al. 2015; Dudin et al. 2019). Being able to study this mechanism of specific gene regulation in more depth could be of relevance to elucidate the initial steps of cell specialization.

On the other hand, investigating the dynamics of cell division during the coenocytic stage of $C$. fragrantissima in depth will help us to understand the similarities and differences with the coenocytes of some animal species' embryos (Figure 3.2d) (de Mendoza et al. 2015; Ondracka et al. 2018).

As a conclusion, $C$. fragrantissima is one of the known ichthyosporeans that could be a more fruitful model organism in the near future for many reasons. First, it is easily cultivated and manipulated in laboratory conditions; second, it presents an apparently linear life cycle and a fairly good description of its different life stages, and third, it has a relatively compact and well-annotated genome, and lastly there is a reasonable availability of genetic tools. All together, this makes $C$. fragrantissima a very good candidate for the study of the evolution of the holozoa clade but also for addressing several open questions concerning the evolution toward multicellularity in animals.

\subsubsection{SPHAEROFORMA ARCTICA}

Although genetic tools are yet to be developed for Sphaeroforma arctica, we thought it important to briefly introduce this organism in this chapter. Recently, two reports have unveiled insightful information on the cellularization and the nuclear division during the coenocytic stage of $S$. arctica (Ondracka et al. 2018; Dudin et al. 2019). These new findings will undoubtedly open new research avenues for all ichthyosporeans, and S. arctica will be considered a good candidate for future studies, especially those addressing questions of general interest for eukaryote biology and evolution.

S. arctica was first isolated from an artic marine amphipod, cultivated in the laboratory and described by Jøstensen and collaborators (2002). The authors also analyzed the chemical composition of its cell wall in order to find specific adaptations to cold water. Its cell wall presents a high content of polyunsaturated fatty acids (more than 70\%), suggesting that they contribute to survival in cold waters (Jøstensen et al. 2002). S. arctica grows in laboratory conditions at $12^{\circ} \mathrm{C}$ in $\mathrm{MB}$ through a linear vegetative life cycle that is completed in approximately 48 hours. Briefly, small round newborn cells proliferate in a multinucleated coenocyte through several rounds of synchronous nuclear divisions, which cellularize at the moment of newborn cell release by bursting from the parental coenocyte (Jøstensen et al. 2002; Ondracka et al. 2018). The absence of alternative stages such as flagellated motile amoebas, budding or hyphal forms makes the $S$. arctica life cycle ideally simple for some studies. In addition, its genome and transcriptome as well as an accurate phylogenetic placement have been obtained for this species (de Mendoza et al. 2015; Torruella et al. 2015).

These features make $S$. arctica an ideal species for further investigations. Indeed, recent studies have unveiled the patterns of cellularization and control of cell division that were previously unknown outside animal lineages. The $S$. arctica cellularization process shares some mechanisms and regulatory pathways with the one present in animals, and it also presents some specific players likely shared with the rest of ichthyosporeans (Figure 3.1) (Dudin et al. 2019). Similarly, detailed studies of nuclear division in S. arctica cultures demonstrated that the timing of nuclear division is not affected by cell size or growth rate and is highly synchronous (Ondracka et al. 2018). This feature distinguishes $S$. arctica from filamentous fungi and more resembles the early divisions of animal embryos.

The main drawback of turning $S$. arctica into a model organism is mainly the difficulty of finding a feasible transfection method. So far, a variety of methods based both on chemical and physical approaches, such as electroporation, lipid-based methods and calcium precipitate protocols, have been tried without success (dx.doi.org/10.17504/protocols. io.z6ef9be). A hard cell wall being already present when the new generation of cells is expelled from the coenocyte is likely the main obstacle to efficiently introducing foreign DNA into the organism. Nevertheless, the fact that new model organisms are now being successfully developed using different strategies is promising for S. arctica to be an experimentally tractable organism in the near future.

\subsection{CORALLOCHYTREA/PLURIFORMEA}

The Corallochytrea clade is also known as Pluriformea because of the great variety of forms exhibited during the life cycles of the organisms composing this lineage (Hehenberger et al. 2017). Corallochytrea is the fourth clade of unicellular Holozoa, a sister-group to Ichthyosporea and in a key phylogenetic position for researchers to study the evolution from unicellular to multicellular organisms (Figure 3.1). To date, this lineage is composed of only two described species: Corallochytrium limacisporum and Syssomonas multiformis (Raghu-kumar 1987; Hehenberger et al. 2017; Tikhonenkov et al. 2020a). Intriguingly, C. limacisporum 
(a) Abeoforma whisleri
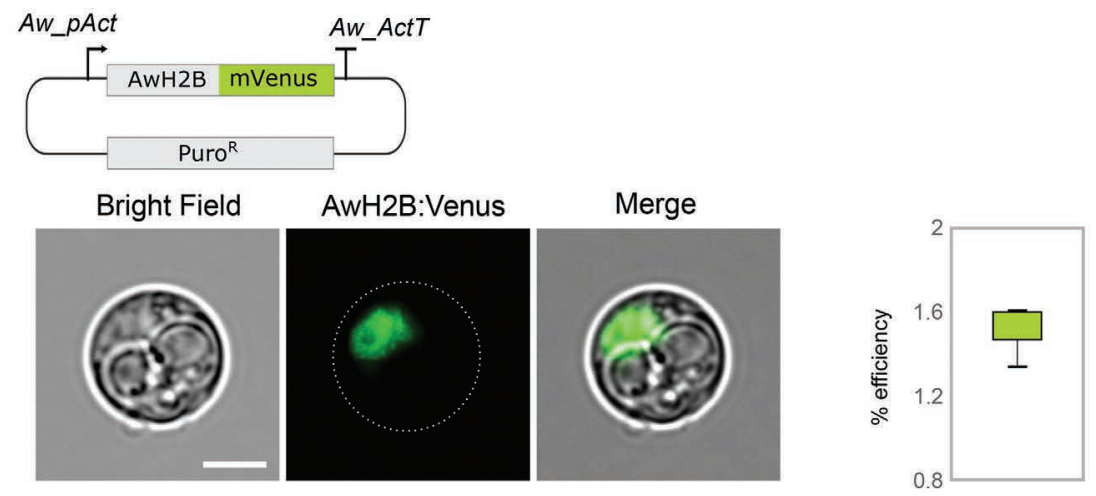

(b) Creolimax fragrantissima

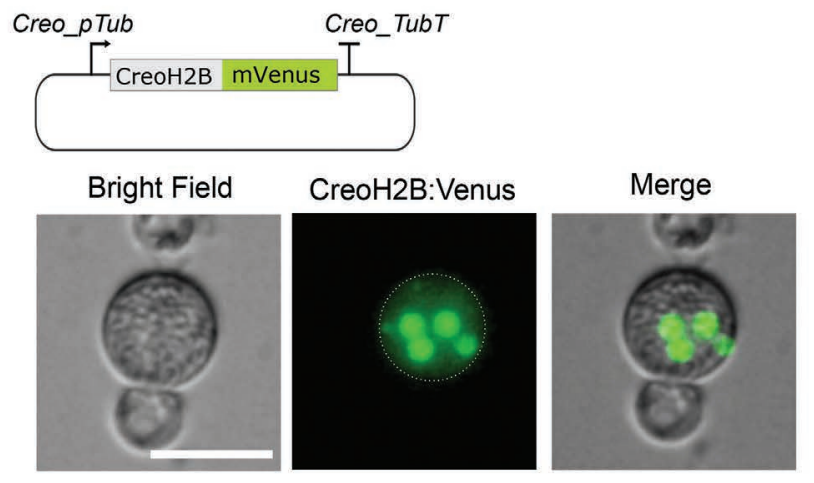

(c) Corallochytrium limacisporum
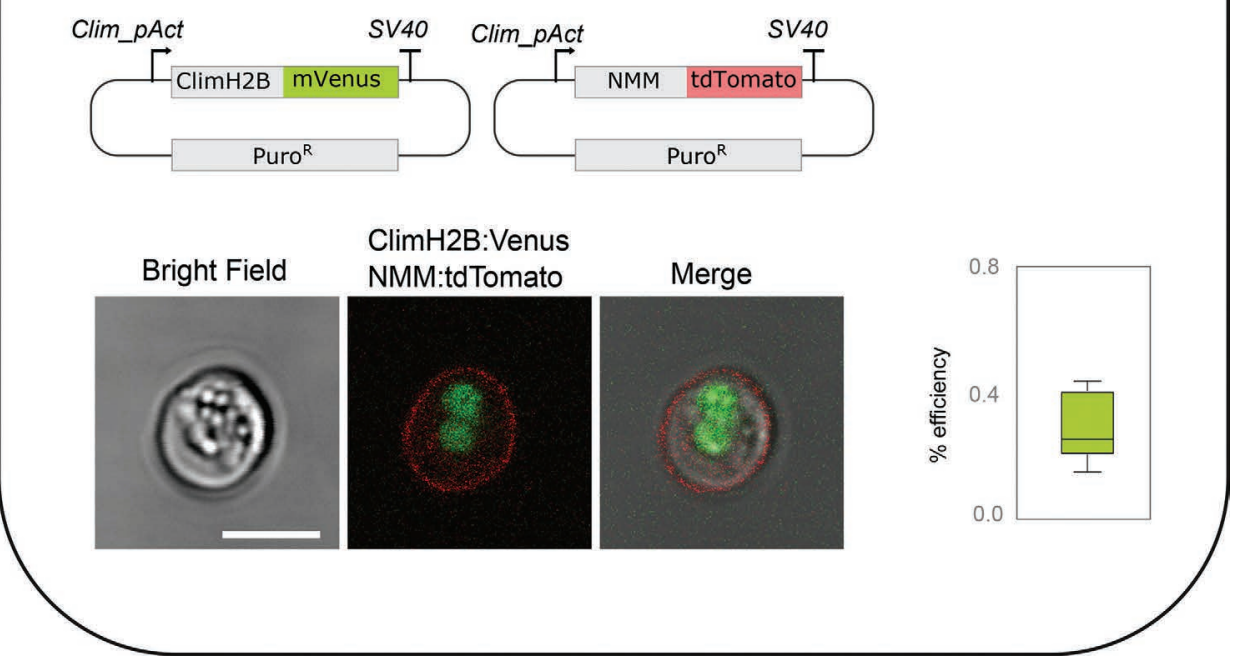

FIGURE 3.4 Live imaging of transfected cells of Abeofroma whisleri, Creolimax fragrantissima and Corallochytrium limacisporum. Images are complemented with diagrams of transfection cassettes. Abeoforma whisleri, nuclei labeling: mVenus fluorescent protein fused to endogenous Histone 2B under the actin promoter and terminator. Creolimax fragrantissima, nuclei labeling: mVenus fluorescent protein fused to endogenous Histone $2 \mathrm{~B}$ under the tubulin promoter and terminator. Corallochytrium limacisporum, nuclei labeling: mVenus fluorescent protein fused to endogenous Histone 2B under the actin promoter and SV40 terminator. Plasma membrane labeling: tdTomato fluorescent protein fused to the endogenous N-myristoylation motif of the src gene (see main text) under the actin promoter and SV40 terminator. Reported transfection efficiency only for Abeoforma whisleri and Coralochytrium limacisporum from our own experiments. Scale bars (a) and (c) $5 \mu \mathrm{m}$, (b) $50 \mu \mathrm{m}$. 
contains a complete flagellar toolkit (Torruella et al. 2015), but its flagellated forms occur sporadically in our culture conditions, whereas in contrast, the most commonly occurring stage of $S$. multiformis consists of flagellated forms (Tikhonenkov et al. 2020a). Both representatives of this clade show some morphological resemblance in their life cycle, S. multiformis being the one with a greater variety of forms. As an example, both organisms have active amoeboid forms and also present complex multicellular stages (Figure 3.2d) (Tikhonenkov et al. 2020a; Kożyczkowska et al. 2021).

In addition to its key phylogenetic position, $C$. limacisporum has many of the desirable features for an organism to be developed as genetically tractable (see next section). On the other hand, unfortunately, cultures of $S$. multiformis are no longer available, and therefore it is difficult to speculate on the possibility of this organism becoming an experimentally treatable organism.

\subsubsection{Corallochytrium limacisporum}

C. limacisporum is a small, marine, free-living corallochytrean isolated from coral reefs of India and Hawaii (Raghukumar 1987). This taxa possesses numerous features that make it an attractive candidate for further functional analysis. It grows very fast and under axenic conditions, and most importantly, it is able to grow in both liquid and agar media, allowing for easy screenings and selection of individual transformed clones. Moreover, it is the only corallochytrean with a completely sequenced and well-annotated genome (Grau-Bové et al. 2017). Finally, besides these technical advantages, $C$. limacisporum has a peculiar and understudied biology, with a complex life cycle and, as we mentioned before, some fungal-like features. For all these reasons, developing genetic tools in this fascinating unicellular organism will for sure be useful for several scientific questions/fields.

\subsubsection{Transfection and Selection}

Different antibiotics, antifungals and herbicides had been tested in C. limacisporum, and the antibiotic puromycin was selected as the most adequate for its efficiency and apparent low toxicity (Kożyczkowska et al. 2021). In addition to selection by antibiotics, it would be ideal to have a double selection system that would also allow us to screen transfected cells by fluorescence microscopy. Therefore, a dual selection system based on resistance to puromycin and mCherry expression was set up. Two recombinant plasmids, CAMP (Corallochytrium Actin Mcherry Pac) and CTMP (Corallochytrium Tubulin Mcherry Pac), were used for optimizing the transfection parameters (see also "Plasmids" section).

Different methods of transfection that had worked for other protists, yeast or eukaryote cells in general based on chemical or physical methods were tested, but only electroporation was successful. Initially, positive results using an in electrode apparatus from Invitrogen, the Neon system, which allows modifying the electric pulse and the duration of the pulse (dx.doi.org/10.17504/protocols.io.hmwb47e), were obtained. Nevertheless, this protocol did not have enough reproducibility to carry out downstream applications, and we selected the electroporator 4D-Nucleofector from Lonza, which was being used with greater efficiency in other protists (Figure 3.3e) (Kożyczkowska et al. 2021 and Protcols.io: dx.doi.org/10.17504/protocols. io.r5ud86w; see sections for $S$. rosetta, A. whisleri and $C$. fragrantissima).

One of the important factors was the cell density and age of the starting culture to maximize efficiency. Similarly to $A$. whisleri, the cells should be washed with 1X PBS to remove the culture media. Co-transfection of highly pure and highly concentrated carrier plasmid DNA (empty pUC19) was another key factor that significantly increased efficiency. In general, some fluorescent cells could be observed after 24 hours post-transfection, although there was always a significant increase in positive cells after 48 hours, after which puromycin was added. In the case of C. limacisporum, the combination of buffer P3 and code EN-138 from the 4D-Nucleofector (Lonza) proved the most optimal for successful transfection (Figure 3.3e) (Kożyczkowska et al. 2021). Clonal lines can be obtained by plating a dilution of the cells in MB agar plates containing puromycin (Kożyczkowska et al. 2021).

As an immediate contribution from these developed genetic tools, the description of the life cycle of C. limacisporum and the unraveling of some unexpected traits, was possible. It has been discovered that $C$. limacisporum has two different paths for cell division, binary fission and coenocytic growth (Figure 3.2d), demonstrating that the $C$. limacisporum life cycle is non-linear and more complex than previously thought (Raghu-kumar 1987). Additionally, some particular features of C. limacisporum not commonly found in eukaryotes were described: first the decoupling of cytokinesis and karyokinesis in binary fission and second the observation of some examples of asynchronous nuclei divisions during coenocytic growth. The possibility to expand functional studies of these features in C. limacisporum will undoubtedly contribute to a better characterization of this unicellular holozoan.

\subsubsection{Plasmids}

As mentioned, a double selection system was engineered. The CAMP plasmid contained the pac gene to provide drug resistance (Luna et al. 1988) and the $m$ Cherry gene to produce fluorescence in the positively transfected cells. In order to drive transcription with endogenous promoters, the upstream non-coding sequence of the actin and tubulin genes from $C$. limacisporum and the 3'UTR terminator of the actin gene from the ichthyosporean $A$. whisleri were cloned in order to avoid homologous recombination at the actin locus. The CAMP and CTMP plasmids were indistinguishable in their phenotype, fluorescent labeling of the cytoplasm in C. limacisporum revealing a "crescent moon-like" shape produced by the presence of a large vacuole that occupies the $65 \%$ of the cell's volume (Kożyczkowska et al. 2021). 
Progress into understanding the cell biology of C. limacisporum (see transfection section) was possible through the generation of constructs tagging sub-cellular components, such as the plasma membrane, cytoskeleton, cytoplasm and nucleus (Kożyczkowska et al. 2021). To construct the pactNMN-tdTomato plasmid, the predicted N-myristoylation motif (NMM) from the $\operatorname{Src}$ tyrosine kinase orthologue (Gene ID Clim_evm93s153) was used. This motif has been successfully used in C. owczarzaki to direct the fusion protein to the plasma membrane (Parra-Acero et al. 2018). Our results show that this motif was also plasma membrane specific in $C$. limacisporum and therefore might also be useful in other organisms (Figure 3.4c) (Kożyczkowska et al. 2021). To visualize the cytoskeleton, the 17-amino acid peptide LifeAct that binds specifically to filamentous actin (ibidi) was fused to the mCherry protein pact-LifeAct. Finally, the construct pact-H2B-mVenus contains the endogenous gene of C. limacisporum (Gene ID Clim_evm20s1) fused to the mVenus fluorescent protein. In addition, the construct contains the actin promoter, with the dual system of puromycin resistance as well as fluorescence (Kożyczkowska et al. 2021).

\subsubsection{Prospects}

The development of specific recombinant plasmids together with stable transfection in $C$. limacisporum has provided insightful information about the biology of this organism while also providing the initial tools to set up functional experiments. Importantly, now $C$. limacisporum provides the opportunity to further investigate which are the factors behind different developmental routes (binary fission or coenocytic growth), as well as a promising model to study the mechanisms behind the decoupling of karyokinesis from cytokinesis and the basis of asynchronous nuclear division.

Besides the previously mentioned advances, developing CRISPR/Cas9 genome editing in C. limacisporum is currently ongoing. The establishment of genome editing in the future will allow us to understand, among others, the possible ancestral role of some genes related to multicellular functions in Metazoa.

\subsection{CONCLUDING REMARKS}

We have here described the most recent advances in the handful of model organisms available among unicellular holozoans (Figure 3.1). These model organisms belong to all four clades of unicellular relatives of animals, constituting a functional platform to experimentally address many of the hypotheses regarding the evolution of genes and cellular features along the Holozoa tree. We are eager to see how evolutionary cell biology will take advantage of all those new emerging model systems to address the function of ancestral genes and protein domains, as well as for the conservation or innovation of cell biological processes.

\section{ACKNOWLEDGMENTS}

We would like to thank Núria Ros-Rocher for critical reading of the manuscript and the Multicellgenome laboratory for ideas and discussions. We would also like to thank the European Research Council (ERC) and the Betty and Gordon Moore foundation for their strategic vision in funding the development of these organisms into experimentally tractable organisms. This work was supported by a European Research Council Consolidator Grant (ERC2012-Co-616960) grant to I.R-T. and a Betty and Gordon Moore "New Genetic tools for Marine Protists", Grant number 4973.01 to E.C and I.R-T.

\section{BIBLIOGRAPHY}

Abedin, M., and N. King. 2010. Diverse Evolutionary Paths to Cell Adhesion. Trends in Cell Biology 20: 734-742.

Ai, H., Henderson, J. N., Remington, S. J., and R. E. Campbell. 2006. Directed Evolution of a Monomeric, Bright and Photostable Version of Clavularia Cyan Fluorescent Protein: Structural Characterization and Applications in Fluorescence Imaging. The Biochemical Journal 400: 531-540.

Alegado, R. A., Brown L. W., Cao S., Dermenjian, R. K., Zuzow, R., Fairclough, S. R., Clardy J., and Nicole King. 2012. A Bacterial Sulfonolipid Triggers Multicellular Development in the Closest Living Relatives of Animals. ELife 1: e00013.

Booth, D. S., and N. King. 2020. Genome Editing Enables Reverse Genetics of Multicellular Development in the Choanoflagellate Salpingoeca Rosetta. Edited by Alejandro Sánchez Alvarado, Patricia J Wittkopp, Margaret A Titus, Iñaki Ruiz-Trillo, and Matthew C Gibson. ELife 9: e56193.

Booth, D. S., Szmidt-Middleton, H., and N. King. 2018. Transfection of Choanoflagellates Illuminates Their Cell Biology and the Ancestry of Animal Septins. Molecular Biology of the Cell 29: 3026-3038.

Burkhardt, P., Grønborg, M., McDonald, K., Sulur, T., Wang, Q., and N. King. 2014. Evolutionary Insights into Premetazoan Functions of the Neuronal Protein Homer. Molecular Biology and Evolution 31: 2342-2355.

Caro, F., Miller, M. G., and J. L DeRisi. 2012. Plate-Based Transfection and Culturing Technique for Genetic Manipulation of Plasmodium falciparum. Malaria Journal 11: 22.

Carr, M., Leadbeater, B. S. C., Hassan, R., Nelson, M., and S. L. Baldauf. 2008. Molecular Phylogeny of Choanoflagellates, the Sister Group to Metazoa. Proceedings of the National Academy of Sciences of the United States of America 105: 16641-16646.

Cavalier-Smith, T. 1998. A Revised Six-Kingdom System of Life. Biological Reviews of the Cambridge Philosophical Society 73: 203-266.

Cavalier-Smith, T., and E. E.-Y. Chao. 2003. Phylogeny of Choanozoa, Apusozoa, and Other Protozoa and Early Eukaryote Megaevolution. Journal of Molecular Evolution 56: $540-563$.

Clark, H. J. 1866. Conclusive Proofs of the Animality of the Ciliate Sponges, and of Their Affinities with the Infusoria Flagellata. American Journal of Science 42 (2): 320-324.

Dayel, M. J., Alegado, R. A., Fairclough, S. R., Levin, T. C., Nichols, S. A., McDonald, K., and N. King. 2011. Cell Differentiation and Morphogenesis in the Colony-Forming Choanoflagellate Salpingoeca rosetta. Developmental Biology 357: 73-82. 
Dolan, J., and B. S. Leadbeater. 2015. The Choanoflagellates: Evolution, Biology, and Ecology. Cambridge University Press, Cambridge, UK. 315 pp. Hardcover, ISBN978-0-52188444-0, \$125. Journal of Eukaryotic Microbiology, June.

Dudin, O., Ondracka, A., Grau-Bové, X., Haraldsen, A. A. B., Toyoda, A., Suga, H., Bråte, J., and I. Ruiz-Trillo. 2019. A Unicellular Relative of Animals Generates a Layer of Polarized Cells by Actomyosin-Dependent Cellularization. ELife 8: e49801. https://doi.org/10.7554/eLife.49801.

Ensenauer, R., Hartl, D., Vockley, J., Roscher, A. A., and U. Fuchs. 2011. Efficient and Gentle SiRNA Delivery by Magnetofection. Biotechnic \& Histochemistry: Official Publication of the Biological Stain Commission 86: 226-231.

Fairclough, S. R., Chen, Z., Kramer, E., Zeng, Q., Young, S., Robertson, H. M., E. Begovic, et al. 2013. Premetazoan Genome Evolution and the Regulation of Cell Differentiation in the Choanoflagellate Salpingoeca rosetta. Genome Biology 14: R15.

Fairclough, S. R., Dayel, M. J., and N. King. 2010. Multicellular Development in a Choanoflagellate. Current Biology: CB 20: R875-R876.

Faktorová, D., Nisbet, E. R., Fernández Robledo, J. A., Casacuberta, E., Sudek L., Allen A. E., M. Ares, et al. 2020. Genetic Tool Development in Marine Protists: Emerging Model Organisms for Experimental Cell Biology. Nature Methods 17: 481-494.

Foster, A. J., Martin-Urdiroz, M., Yan, X., Wright, H. S., Soanes, D. M., and N. J. Talbot. 2018. CRISPR-Cas9 RibonucleoproteinMediated Co-Editing and Counterselection in the Rice Blast Fungus. Scientific Reports 8: 14355.

Gaudet, P., Pilcher, K. E., Fey, P., and R. L. Chisholm. 2007. Transformation of Dictyostelium Discoideum with Plasmid DNA. Nature Protocols 2: 1317-1324.

Graham, F. L., and A. J. van der Eb. 1973. A New Technique for the Assay of Infectivity of Human Adenovirus 5 DNA. Virology 52: 456-467.

Grau-Bové, X., Torruella, G., Donachie, S., Suga, H., Leonard, G., Richards, T. A., and I. Ruiz-Trillo. 2017. Dynamics of Genomic Innovation in the Unicellular Ancestry of Animals. Edited by Diethard Tautz. ELife 6 (July): e26036.

Grosjean, F., Bertschinger, M., Hacker, D. L., and F. M. Wurm. 2006. Multiple Glycerol Shocks Increase the Calcium Phosphate Transfection of Non-Synchronized CHO Cells. Biotechnology Letters 28: 1827-1833.

Guo, L., Wang, L., Yang, R., Feng, R., Li, Z., Zhou, X., Z. Dong, et al. 2017. Optimizing Conditions for Calcium Phosphate Mediated Transient Transfection. Saudi Journal of Biological Sciences, Computational Intelligence Research \& Approaches in Bioinformatics and Biocomputing 24: 622-629.

Han, H. A., Sheng Pang, J. K., and B.-S. Soh. 2020. Mitigating Off-Target Effects in CRISPR/Cas9-Mediated In Vivo Gene Editing. Journal of Molecular Medicine 98: 615-632.

Hehenberger, E., Tikhonenkov, D. V., Kolisko, M., del Campo, J., Esaulov, A. S., Mylnikov, A. P., and P. J. Keeling. 2017. Novel Predators Reshape Holozoan Phylogeny and Reveal the Presence of a Two-Component Signaling System in the Ancestor of Animals. Current Biology 27: 2043-2050.

Hoffmeyer, T. T., and P. Burkhardt. 2016. Choanoflagellate Models: Monosiga brevicollis and Salpingoeca rosetta. Current Opinion in Genetics \& Development, Developmental Mechanisms, Patterning and Evolution 39: 42-47.

Jacobs, J. Z., Ciccaglione, K. M., Tournier, V., and M. Zaratiegui. 2014. Implementation of the CRISPR-Cas9 System in Fission Yeast. Nature Communications 5: 5344.
Janse, C. J., Franke-Fayard, B., Mair, G. R., Ramesar, J., Thiel, C., Engelmann, S., Matuschewski, K., van Gemert, G. J., Sauerwein, R. W., and A. P. Waters. 2006. High Efficiency Transfection of Plasmodium Berghei Facilitates Novel Selection Procedures. Molecular and Biochemical Parasitology 145: 60-70.

Jiang, W., Brueggeman, A., Horken, K., Plucinak, T., and D. Weeks. 2014. Successful Transient Expression of Cas9 and Single Guide RNA Genes in Chlamydomonas reinhardtii. Eukaryotic Cell 13.

Jordan, M., and F. Wurm. 2004. Transfection of Adherent and Suspended Cells by Calcium Phosphate. Methods (San Diego, Calif.) 33: 136-143.

Jøstensen, J.-P., Sperstad, S., Johansen, S., and B. Landfald. 2002. Molecular-Phylogenetic, Structural and Biochemical Features of a Cold-Adapted, Marine Ichthyosporean Near the Animal-Fungal Divergence, Described from In Vitro Cultures. European Journal of Protistology 38: 93-104.

Kim, J. A., Cho, K., Shin, M. S., Lee, W. G., Jung, N., Chung, C., and J. Keun Chang. 2008. A Novel Electroporation Method Using a Capillary and Wire-Type Electrode. Biosensors \& Bioelectronics 23: 1353-1360.

Kim, S. K., Kim, D., Woo Cho, S., Kim, J., and J.-S. Kim. 2014. Highly Efficient RNA-Guided Genome Editing in Human Cells via Delivery of Purified Cas9 Ribonucleoproteins. Genome Research 24.

Kim, S. K., Shindo, A., Park, T. J., Oh, E. C., Ghosh, S., Gray, R. S., R. A. Lewis, et al. 2010. Planar Cell Polarity Acts through Septins to Control Collective Cell Movement and Ciliogenesis. Science (New York, N.Y.) 329: 1337-1340.

King, N. 2005. Choanoflagellates. Current Biology: $C B$ 15: R113-R114.

King, N., Hittinger, C. T., and S. B. Carroll. 2003. Evolution of Key Cell Signaling and Adhesion Protein Families Predates Animal Origins. Science (New York, N.Y.) 301: 361-363.

King, N., Westbrook, M. J., Young, S. L., Kuo, A., Abedin, M., Chapman, J., S. Fairclough, et al. 2008. The Genome of the Choanoflagellate Monosiga brevicollis and the Origin of Metazoans. Nature 451: 783-788.

Kożyczkowska, A., Najle, S. R., Ocaña-Pallarès, E., Aresté, C., Shabardina, V., Ara, P. S., Ruiz-Trillo, I., and E. Casacuberta. 2021. Stable Transfection in the Protist Corallochytrium limacisporum Allows Identification of Novel Cellular Features Among Unicellular Relatives of Animals. Current Biology 31: 1-7. https://doi.org/10.1016/j.cub.2021. 06.061

Laundon, D., Larson, B. T., McDonald, K., King, N., and P. Burkhardt. 2019. The Architecture of Cell Differentiation in Choanoflagellates and Sponge Choanocytes. PLoS Biology 17 (4): e3000226.

Leadbeater, B. S. C., Yu, Q., Kent, J., and D. J. Stekel. 2009. Three-Dimensional Images of Choanoflagellate Loricae. Proceedings of the Royal Society B: Biological Sciences 276: 3-11.

Levin, T. C., Greaney A. J., Wetzel, L., and N. King. 2014. The Rosetteless Gene Controls Development in the Choanoflagellate $S$. rosetta. ELife 3 (October).

Levin, T. C., and N. King. 2013. Evidence for Sex and Recombination in the Choanoflagellate Salpingoeca rosetta. Current Biology 23: 2176-2180.

Liang, X., Potter, J., Kumar, S., Zou, Y., Quintanilla, R., Sridharan, M., J. Carte, et al. 2015. Rapid and Highly Efficient Mammalian Cell Engineering via Cas9 Protein Transfection. Journal of Biotechnology 208 (August): 44-53. 
López-Escardó, D., Grau-Bové, X., Guillaumet-Adkins, A., Gut, M., Sieracki, M. E., and I. Ruiz-Trillo. 2019. Reconstruction of Protein Domain Evolution Using Single-Cell Amplified Genomes of Uncultured Choanoflagellates Sheds Light on the Origin of Animals. Philosophical Transactions of the Royal Society B: Biological Sciences 374 (1786): 20190088.

Luna, S. de la, Soria, I., Pulido, D., Ortín, J., and A. Jiménez. 1988. Efficient Transformation of Mammalian Cells with Constructs Containing a Puromycin-Resistance Marker. Gene 62: 121-126.

Mah, J. L., Christensen-Dalsgaard, K. K., and S. P. Leys. 2014. Choanoflagellate and Choanocyte Collar-Flagellar Systems and the Assumption of Homology. Evolution \& Development 16: 25-37.

Maldonado, M. 2004. Choanoflagellates, Choanocytes, and Animal Multicellularity. Invertebrate Biology 123: 1-22.

Marshall, W. L., and M. L. Berbee. 2011. Facing Unknowns: Living Cultures (Pirum gemmata Gen. Nov., Sp. Nov., and Abeoforma whisleri, Gen. Nov., Sp. Nov.) from Invertebrate Digestive Tracts Represent an Undescribed Clade within the Unicellular Opisthokont Lineage Ichthyosporea (Mesomycetozoea). Protist 162: 33-57.

Marshall, W. L., Celio, G., McLaughlin, D. J., and M. L. Berbee. 2008. Multiple Isolations of a Culturable, Motile Ichthyosporean (Mesomycetozoa, Opisthokonta), Creolimax fragrantissima n. Gen., n. Sp., from Marine Invertebrate Digestive Tracts. Protist 159: 415-433.

Mendoza, A. de, and A. Sebé-Pedrós. 2019. Origin and Evolution of Eukaryotic Transcription Factors. Current Opinion in Genetics \& Development 58-59: 25-32.

Mendoza, A. de, Suga, H., Permanyer, J., Irimia, M., and I. Ruiz-Trillo. 2015. Complex Transcriptional Regulation and Independent Evolution of Fungal-Like Traits in a Relative of Animals. Edited by Alejandro Sánchez Alvarado. ELife 4: e08904.

Mendoza, L., Taylor, J. W., and L. Ajello. 2002. The Class Mesomycetozoea: A Heterogeneous Group of Microorganisms at the Animal-Fungal Boundary. Annual Review of Microbiology 56: 315-344.

Miller, D. J., and R. L. Ax. 1990. Carbohydrates and Fertilization in Animals. Molecular Reproduction and Development 26: 184-198.

Mylnikov, A. P., Tikhonenkov, D. V., Karpov, S. A., and C. Wylezich. 2019. Microscopical Studies on Ministeria vibrans Tong, 1997 (Filasterea) Highlight the Cytoskeletal Structure of the Common Ancestor of Filasterea, Metazoa and Choanoflagellata. Protist 170: 385-396.

Nagai, T., Ibata, K., Sun Park, E., Kubota, M., Mikoshiba, K., and A. Miyawaki. 2002. A Variant of Yellow Fluorescent Protein with Fast and Efficient Maturation for Cell-Biological Applications. Nature Biotechnology 20: 87-90.

Naumann, B., and P. Burkhardt. 2019. Spatial Cell Disparity in the Colonial Choanoflagellate Salpingoeca rosetta. Frontiers in Cell and Developmental Biology 7.

Neufeld, T. P., and G. M. Rubin. 1994. The Drosophila Peanut Gene Is Required for Cytokinesis and Encodes a Protein Similar to Yeast Putative Bud Neck Filament Proteins. Cell 77: 371-379.

Nielsen, C. 2008. Six Major Steps in Animal Evolution: Are We Derived Sponge Larvae? Evolution \& Development 10: 241-257.

Ondracka, A., Dudin, O., and I. Ruiz-Trillo. 2018. Decoupling of Nuclear Division Cycles and Cell Size during the Coenocytic Growth of the Ichthyosporean Sphaeroforma arctica. Current Biology 28: 1964-1969.
Owczarzak, A., Stibbs, H. H., and C. J. Bayne. 1980a. The Destruction of Schistosoma mansoni Mother Sporocysts In Vitro by Amoebae Isolated from Biomphalaria glabrata: An Ultrastructural Study. Journal of Invertebrate Pathology 35: 26-33.

Owczarzak, A., Stibbs, H. H., and C. J. Bayne. 1980b. The Destruction of Schistosoma mansoni Mother Sporocysts In Vitro by Amoebae Isolated from Biomphalaria glabrata: An Ultrastructural Study. Journal of Invertebrate Pathology 35: 26-33.

Paps, J., Medina-Chacón, L. A., Marshall, W., Suga, H., and I. RuizTrillo. 2013. Molecular Phylogeny of Unikonts: New Insights into the Position of Apusomonads and Ancyromonads and the Internal Relationships of Opisthokonts. Protist 164: 2-12.

Parra-Acero, H., Harcet, M., Sánchez-Pons, N., Casacuberta, E., Brown, N. H., Dudin, O., and I. Ruiz-Trillo. 2020. IntegrinMediated Adhesion in the Unicellular Holozoan Capsaspora owczarzaki. Current Biology 30: 4270-4275.

Parra-Acero, H., Ros-Rocher, N., Perez-Posada, A., Kożyczkowska, A., Sánchez-Pons, N., Nakata, A., Suga, H., Najle, S. R., and I. Ruiz-Trillo. 2018. Transfection of Capsaspora owczarzaki, a Close Unicellular Relative of Animals. Development (Cambridge, England) 145 (10).

Pekkarinen, M. 2003. Phylogenetic Position and Ultrastructure of Two Dermocystidium Species (Ichthyosporea) from the Common Perch (Perca fluviatilis). Acta Protozoologica 42 (4): 287-307.

Pettitt, M. E., Orme, B. A. A., Blake, J. R., and B. S. C. Leadbeater. 2002. The Hydrodynamics of Filter Feeding in Choanoflagellates. European Journal of Protistology 38: 313-332.

Raghu-kumar, S. 1987. Occurrence of the Thraustochytrid, Corallochytrium Limacisporum Gen. et Sp. Nov. in the Coral Reef Lagoons of the Lakshadweep Islands in the Arabian Sea. Botanica Marina 30 (1).

Richter, D. J., Fozouni, P., Eisen, M. B., and N. King. 2018. Gene Family Innovation, Conservation and Loss on the Animal Stem Lineage. Edited by Maximilian J Telford. ELife 7: e34226.

Riedl, J., Crevenna, A. H., Kessenbrock, K., Haochen Yu, J., Neukirchen, D., Bista, M., F. Bradke, et al. 2008. Lifeact: A Versatile Marker to Visualize F-Actin. Nature Methods 5: 605.

Ryan, T. J., and S. G. N. Grant. 2009. The Origin and Evolution of Synapses. Nature Reviews Neuroscience 10: 701-712.

Sebé-Pedrós, A., Ballaré, C., Parra-Acero, H., Chiva, C., Tena, J. J., Sabidó, E., Gómez-Skarmeta, J. L., Di Croce, L., and I. Ruiz-Trillo. 2016. The Dynamic Regulatory Genome of Capsaspora and the Origin of Animal Multicellularity. Cell 165: 1224-1237.

Sebé-Pedrós, A., Degnan, B. M., and I. Ruiz-Trillo. 2017. The Origin of Metazoa: A Unicellular Perspective. Nature Reviews Genetics 18: 498-512.

Sebé-Pedrós, A., Irimia, M., Del Campo, J., Parra-Acero, H., Russ, C., Nusbaum, C., Blencowe, B. J., and I. Ruiz-Trillo. 2013. Regulated Aggregative Multicellularity in a Close Unicellular Relative of Metazoa. ELife 2 (December): e01287.

Shalchian-Tabrizi, K., Minge, M. A., Espelund, M., Orr, R., Ruden, T., Jakobsen, K. S., and T. Cavalier-Smith. 2008. Multigene Phylogeny of Choanozoa and the Origin of Animals. PLoS One 3 (5).

Shaner, N. C., Campbell, R. E., Steinbach, P. A., Giepmans, B. N. G., Palmer, A. E., and R. Y. Tsien. 2004. Improved Monomeric Red, Orange and Yellow Fluorescent Proteins 
Derived from Discosoma Sp. Red Fluorescent Protein. Nature Biotechnology 22: 1567-1572.

Shin, S.-E., Lim, J.-M., Koh, H. G., Kim, E. K., Kang, N. K., Jeon, S., S. Kwon, et al. 2016. CRISPR/Cas9-Induced Knockout and Knock-in Mutations in Chlamydomonas reinhardtii. Scientific Reports 6: 27810.

Sigal, C. T., Zhou, W., Buser, C. A., McLaughlin, S., and M. D. Resh. 1994. Amino-Terminal Basic Residues of Src Mediate Membrane Binding through Electrostatic Interaction with Acidic Phospholipids. Proceedings of the National Academy of Sciences of the United States of America 91: 12253-12257.

Singh, R. K., Liang, D., Gajjalaiahvari, U. R., Miquel Kabbaj, M.-H., Paik, J., and A. Gunjan. 2010. Excess Histone Levels Mediate Cytotoxicity via Multiple Mechanisms. Cell Cycle 9: 4236-4244.

Sogabe, S., Hatleberg, W. L., Kocot, K. M., Say, T. E., Stoupin, D., Roper, K. E., Fernandez-Valverde, S. L., Degnan, S. M., and B. M. Degnan. 2019. Pluripotency and the Origin of Animal Multicellularity. Nature 570: 519-522.

Spiliotis, E. T., Hunt, S. J., Hu, Q., Kinoshita, M., and W. J. Nelson. 2008. Epithelial Polarity Requires Septin Coupling of Vesicle Transport to Polyglutamylated Microtubules. The Journal of Cell Biology 180: 295-303.

Stibbs, H. H., Owczarzak, A., Bayne, C. J., and P. DeWan. 1979. Schistosome Sporocyst-Killing Amoebae Isolated from Biomphalaria glabrata. Journal of Invertebrate Pathology 33: $159-170$.

Suga, H., Dacre, M., de Mendoza, A., Shalchian-Tabrizi, K., Manning, G., and I. Ruiz-Trillo. 2012. Genomic Survey of Premetazoans Shows Deep Conservation of Cytoplasmic Tyrosine Kinases and Multiple Radiations of Receptor Tyrosine Kinases. Science Signaling 5 (222): ra35.

Suga, H., and I. Ruiz-Trillo. 2013. Development of Ichthyosporeans Sheds Light on the Origin of Metazoan Multicellularity. Developmental Biology 377: 284-292.

Suga, H., Chen, Z., de Mendoza, A., Sebé-Pedrós, A., Brown, M. W., Kramer, E., M. Carr, et al. 2013. The Capsaspora Genome
Reveals a Complex Unicellular Prehistory of Animals. Nature Communications 4 (1): 2325. https://doi.org/10.1038/ ncomms 3325 .

Tikhonenkov, D. V., Hehenberger, E., Esaulov, A. S., Belyakova, O. I., Mazei, Y. A., Mylnikov, A.P., and P. J. Keeling. 2020a. Insights into the Origin of Metazoan Multicellularity from Predatory Unicellular Relatives of Animals. BMC Biology 18: 39.

Tikhonenkov, D. V., Mikhailov, K. V., Hehenberger, E., Karpov, S. A., Prokina, K. I., Esaulov, A. S., O. I. Belyakova, et al. 2020b. New Lineage of Microbial Predators Adds Complexity to Reconstructing the Evolutionary Origin of Animals. Current Biology 30: 4500-4509.

Tong, S. M. 1997. Heterotrophic Flagellates and Other Protists from Southampton Water, U.K. Ophelia 47: 71-131.

Torruella, G., de Mendoza, A., Grau-Bové, X., Antó, M., Chaplin, M. A., del Campo, J., L. Eme, et al. 2015. Phylogenomics Reveals Convergent Evolution of Lifestyles in Close Relatives of Animals and Fungi. Current Biology 25: 2404-2410.

Torruella, G., Derelle, R., Paps, J., Lang, B. F., Roger, A. J., Shalchian-Tabrizi, K., and I. Ruiz-Trillo. 2012. Phylogenetic Relationships within the Opisthokonta Based on Phylogenomic Analyses of Conserved Single-Copy Protein Domains. Molecular Biology and Evolution 29: 531-544.

Wetzel, L. A., Levin, T. C., Hulett, R. E., Chan, D., King, G. A., Aldayafleh, R., Booth, D. S., Abedin Sigg, M., and N. King. 2018. Predicted Glycosyltransferases Promote Development and Prevent Spurious Cell Clumping in the Choanoflagellate S. rosetta. Edited by Alejandro Sánchez Alvarado and Marianne E Bronner. ELife 7: e41482.

Woznica, A., Cantley, A. M., Beemelmanns, C., Freinkman, E., Clardy, J., and N. King. 2016. Bacterial Lipids Activate, Synergize, and Inhibit a Developmental Switch in Choanoflagellates. Proceedings of the National Academy of Sciences of the United States of America 113: 7894-7899.

Woznica, A., Gerdt, J. P., Hulett, R. E., Clardy, J., and N. King. 2017. Mating in the Closest Living Relatives of Animals Is Induced by a Bacterial Chondroitinase. Cell 170: 1175-1183. 